\title{
Coarse, intermediate and high resolution numerical simulations of the transition of a tropical wave critical layer to a tropical storm
}

\author{
M. T. Montgomery ${ }^{1}$, Z. Wang ${ }^{3}$, and T. J. Dunkerton ${ }^{2,1}$ \\ ${ }^{1}$ Naval Postgraduate School, Monterey CA, USA \\ ${ }^{2}$ NorthWest Research Associates, Bellevue WA, USA \\ ${ }^{3}$ Department of Atmospheric Sciences, University of Illinois, Urbana, IL, USA
}

Received: 14 March 2009 - Published in Atmos. Chem. Phys. Discuss.: 8 December 2009

Revised: 29 August 2010 - Accepted: 9 September 2010 - Published: 18 November 2010

\begin{abstract}
Recent work has hypothesized that tropical cyclones in the deep Atlantic and eastern Pacific basins develop from within the cyclonic Kelvin cat's eye of a tropical easterly wave critical layer located equatorward of the easterly jet axis. The cyclonic critical layer is thought to be important to tropical cyclogenesis because its cat's eye provides (i) a region of cyclonic vorticity and weak deformation by the resolved flow, (ii) containment of moisture entrained by the developing flow and/or lofted by deep convection therein, (iii) confinement of mesoscale vortex aggregation, (iv) a predominantly convective type of heating profile, and (v) maintenance or enhancement of the parent wave until the developing proto-vortex becomes a self-sustaining entity and emerges from the wave as a tropical depression. This genesis sequence and the overarching framework for describing how such hybrid wave-vortex structures become tropical depressions/storms is likened to the development of a marsupial infant in its mother's pouch, and for this reason has been dubbed the "marsupial paradigm".

Here we conduct the first multi-scale test of the marsupial paradigm in an idealized setting by revisiting the Kurihara and Tuleya problem examining the transformation of an easterly wave-like disturbance into a tropical storm vortex using the WRF model. An analysis of the evolving winds, equivalent potential temperature, and relative vertical vorticity is presented from coarse $(28 \mathrm{~km})$, intermediate $(9 \mathrm{~km})$ and high resolution $(3.1 \mathrm{~km})$ simulations. The results are found to support key elements of the marsupial paradigm by demonstrating the existence of a rotationally dominant region with minimal strain/shear deformation near the center of the critical
\end{abstract}

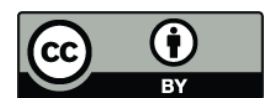

Correspondence to: M. T. Montgomery (mtmontgo@nps.edu) layer pouch that contains strong cyclonic vorticity and high saturation fraction. This localized region within the pouch serves as the "attractor" for an upscale "bottom up" development process while the wave pouch and proto-vortex move together.

Implications of these findings are discussed in relation to an upcoming field experiment for the most active period of the Atlantic hurricane season in 2010 that is to be conducted collaboratively between the National Oceanic and Atmospheric Administration (NOAA), the National Science Foundation (NSF), and the National Aeronautics and Space Adminstration (NASA).

\section{Introduction}

The genesis of tropical cyclones has been regarded, until very recently, as one of the "great mysteries of the tropical atmosphere" (Emanuel, 2005). Like a steep unconquered peak in the Himalaya, for which a tractable route of ascent remains to be discovered, the genesis problem presents a number of scientific and technical challenges from observational, theoretical and numerical directions. The multi-scale nature of the problem has been arguably the most daunting crux of the climb. We believe that a successful summit bid requires a quantum leap in dynamical understanding of the Lagrangian nature of the genesis process and that such insight will open new routes to the top.

Building on previous work examining tropical easterly wave lifecycles (e.g. Thorncroft and Hoskins, 1994) and upscale organizational processes by vortical hot towers (VHTs) within such favorable environments (Montgomery et al., 2006), recent work by Dunkerton et al. (2009; hereafter

Published by Copernicus Publications on behalf of the European Geosciences Union. 
DMW09) proposed a new paradigm for tropical cyclogenesis that recognizes the intrinsic multiscale nature of the problem from synoptic, sub-synoptic, mesoscale and cloud scales. Using three independent datasets, ECMWF Reanalysis data, TRMM 3B42 3-h precipitation and best track data from the National Hurricane Center (NHC), the Kelvin cat's eye within the critical layer of a tropical easterly wave was hypothesized to be important to tropical storm formation because: H1) wave breaking or roll-up of cyclonic vorticity and lower-tropospheric moisture near the critical surface in the lower troposphere provides a favorable environment for the aggregation of vorticity seedlings for TC formation; (H2) the cat's eye is a region of approximately closed circulation, where air is repeatedly moistened by deep moist convection and protected to some degree from dry air intrusion; (H3) the parent wave is maintained and possibly enhanced by diabatically amplified mesoscale vortices within the wave. This genesis sequence and the overarching framework for describing how such hybrid wave-vortex structures become tropical depressions is likened to the development of a marsupial infant in its mother's pouch wherein the juvenile protovortex is carried along by the mother parent wave until it is strengthened into a self-sustaining entity. A survey of 55 named storms in the Atlantic and eastern Pacific sectors during August-September 1998-2001 was shown to support this so-called "marsupial paradigm". Tropical cyclogenesis tended to occur near the intersection of the trough axis and the critical surface of the wave, the nominal center of the cat's eye.

The marsupial paradigm provides a useful road map for exploration of synoptic-mesoscale linkages essential to tropical cyclogenesis. In this paper we begin a systematic test of the marsupial paradigm by revisiting the canonical problem of the transformation of an idealized easterly wave-like disturbance into a tropical storm vortex. Both coarse resolution (cloud parameterization) and high resolution (cloud representing) numerical experiments are conducted, and the high-resolution experiment includes a region of intermediate horizontal resolution in order to compare, side-by-side, two otherwise identical storms with near cloud-resolving numerics. These experiments are a first step towards closing the gap between observationallly resolvable scales (synoptic, meso-alpha) and infrequently sampled cloud-system and cloud-scale process (meso-beta and meso-gamma, including VHTs)

\section{Model and experiment design}

\subsection{WRF model}

The numerical model used is the Weather Research and Forecasting (WRF) model, developed and maintained by NCAR (Skamarock et al., 2005). The WRF model is a fully compressible, Euler nonhydrostatic model with a run-time hy- drostatic option available. It adopts Arakawa C-grid staggering to facilitate the natural discretization of the continuity and tracer equations. Time-split integration uses a 3rdorder Runge-Kutta scheme with smaller steps for acoustic and gravity wave modes, and 2nd to 6th order advection options are used in the horizontal and vertical discretizations in the form of flux divergence. The model uses a terrainfollowing, hydrostatic-pressure vertical coordinate, which is also called a "mass vertical coordinate".

Our WRF implementation employs the nonhydrostatic option and possesses 33 vertical levels with 23 of them below $500 \mathrm{hPa}$. Symmetric lateral boundary conditions are imposed at the north-south boundaries, and periodic boundary conditions are used in the west-east direction. Standard "physics" packages are employed for the parameterization of deep and shallow convection (Betts-Miller-Janjic scheme), microphysics (Kessler scheme), PBL (Yonsei University scheme), longwave radiation (RRTM scheme) and shortwave radiation (Dudhia scheme).

\subsection{Experimental design}

\subsubsection{Basic state flow}

Our objective is to test the hypothesized role of the critical surface and its associated finite-amplitude cat's eye flow in the ensuing multi-scale convective evolution within and near the easterly wave's pouch. To help provide a benchmark for future studies, the now classical tropical cyclone genesis experiment performed by Kurihara and Tuleya (1981; referred to hereafter as KT81) will be re-conducted here and interpreted with new eyes using the marsupial paradigm of DMW09.

We adopt the same basic state flow as in KT81. The initial basic flow is defined as follows:

$\left\{\begin{array}{l}\bar{u}(\phi, \sigma)=u_{1} \cos (\sigma \pi) \tanh \left(\frac{\phi-\phi_{0}}{D}\right)+u_{0} \frac{\cos \phi}{\cos \phi_{0}} \\ \bar{v}=0\end{array}\right.$

where $\sigma=\frac{p}{p_{*}}, p_{*}$ is the surface pressure, $\bar{u}$ and $\bar{v}$ denote the zonal and meridional flow of the base state, respectively, $\phi$ denotes latitude, $\phi_{0}=18^{\circ} \mathrm{N}$ is the center latitude of the zonal channel, and $D=2.5^{\circ}, u_{1}=+3.75 \mathrm{~m} \mathrm{~s}^{-1}$ and $u_{0}=-5 \mathrm{~m} \mathrm{~s}^{-1}$. This idealized zonal flow is consistent with the observed zonal flow during Phase III of GATE near $80^{\circ} \mathrm{W}$ (and consistent also with recent NCAR re-analyses for this region between 1968-1998 as reported in Fig. 1 of Hall et al., 2006). The main difference between this model configuration and that of KT81 is that the channel width is extended to $50^{\circ}$ latitude in order to reduce the influence of the lateral boundary. Following KT81, the mean surface pressure, temperature and geopotential are obtained from the basic flow by inverting the geostrophic and hydrostatic balance equations in Cartesian coordinates (see Appendix A for details).

As shown in Fig. 1, an easterly mean flow is prevalent throughout the model domain, and a broad easterly jet is 

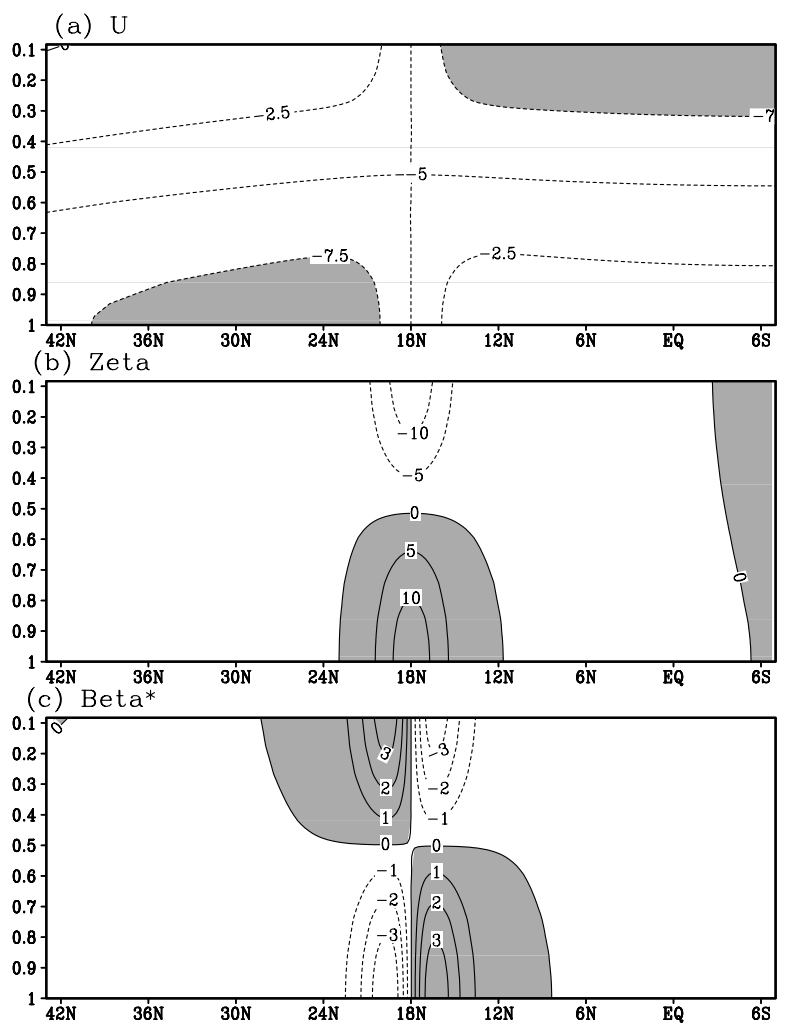

Fig. 1. Latitude-height distributions of the idealized basic zonal flow (top, $\mathrm{ms}^{-1}$ ), the corresponding relative vorticity (middle, $10^{-6} \mathrm{~s}^{-1}$ ) and the meridional gradient of the relative vorticity (bottom, $10^{-11} \mathrm{~m}^{-1} \mathrm{~s}^{-1}$ ). The ordinate is $\sigma$ and the abscissa is latitude.

present in the northern part of the domain. The horizontal shear of the zonal flow is largely confined within $\sim 400 \mathrm{~km}$ on both sides of the center latitude $\left(18^{\circ} \mathrm{N}\right)$. North of the center latitude, the basic flow has a westerly vertical shear; south of the center latitude, the basic flow has an easterly vertical shear of similar magnitude. Along the center latitude, the basic zonal flow is $-5 \mathrm{~m} \mathrm{~s}^{-1}$ and is constant with height (no vertical shear). Since the meridional gradient of the relative vorticity changes sign at the center latitude in both the upper and the lower troposphere, it satisfies the (Rayleigh) necessary condition for barotropic instability (Holton, 1992). For simplicity, the calculations here are carried out on an f-plane (corresponding to $18^{\circ} \mathrm{N}$ ). (When the beta effect is included, the location where the meridional gradient of the absolute vorticity changes sign is slightly north of the center latitude).

\subsubsection{Unstable modes and initial disturbances}

To determine the most unstable (dry) inviscid mode for this zonal mean flow, small amplitude random noise to the initial wind field was introduced, and the model was integrated forward in time without moist processes or surface friction. As is usual, with a periodic boundary condition in the east- west direction, the zonal scale of the domain constrains the allowed wavelength of the unstable modes (the zonal scale of the domain is an integer multiple of the wavelength of any wave it resolves). So as to permit the excitation of a sufficiently broad spectrum the zonal domain is set to $100^{\circ}$ longitude. To reduce computation time, the horizontal grid spacing was set to $56 \mathrm{~km}$. The initial disturbance was produced by adding random wind noise at each grid point with maximum magnitude about $0.25 \mathrm{~m} \mathrm{~s}^{-1}$ (equivalent to random relative vertical vorticity disturbances of order $10^{-5} \mathrm{~s}^{-1}$ ). The magnitude of the wind noise, $A$, is taken to be independent of height, but decays exponentially away from the center latitude and is confined within $8.5^{\circ}$ on both sides of the center latitude (Fig. 2a) according to the formula:

$A=\left\{\begin{array}{l}A_{0}\left[\exp \left(-\frac{9}{2}\left(\frac{y}{L_{y}}\right)^{2}\right)-\exp \left(-\frac{9}{2}\right)\right] \text { if }\left|\frac{y}{L_{y}}\right|<1 \\ 0 \text { otherwise }\end{array}\right.$

where $L_{y}=952 \mathrm{~km}\left(8.5^{\circ}\right), A_{0}=0.25 \mathrm{~m} \mathrm{~s}^{-1}$, and $y$ is zero at the center latitude $\left(18^{\circ} \mathrm{N}\right)$, where the meridional gradient of the relative vorticity changes sign.

After 45 days of integration two wave groups dominate the response. The first group is confined in the lower troposphere (below $500 \mathrm{mb}$ ) and the maximum perturbation winds reside near the lower surface (Fig. 2b). The second group of waves is confined in the upper troposphere (not shown), with a similar magnitude and a longer wavelength $(\sim 3700 \mathrm{~km})$. By 35 days the perturbation meridional velocity magnitude at the surface increases to approximately $1 \mathrm{~m} \mathrm{~s}^{-1}$, and the wavelength is approximately $25^{\circ}$ longitude (about $2800 \mathrm{~km}$ ). As shown in Fig. 3, the e-folding time of this lower-tropospheric mode is approximately 9.8 days, but it takes approximately 30 days, including the incubation period, for the random perturbations to develop into a coherent wave train pattern. As is evident from Fig. 2b, these waves tilt SW-NE against the prevailing latitudinal shear during their growth phase, suggesting a barotropic instability. After 45 days of integration( Figs. 2c and 3), the growth of the dominant mode in the lower troposphere has ceased and the mode has reached a saturation amplitude for the meridional wind of approximately $1.8 \mathrm{~m} \mathrm{~s}^{-1}$. The numerical model conserves the corresponding local extremum of vorticity reasonably well at subsequent times (not shown). From Fig. 2c we see that the flow evolution has transformed the initial vortex strip into a train of (cyclonic) Kelvin cat's eyes with maximum cyclonic vorticity inside each closed "gyre-pouch".

Given the slow development of dry waves in the KT81 basic state, we conclude that this basic flow supports only a marginally unstable mode. The zonal phase speed of this marginally unstable wave is found to be approximately $-5 \mathrm{~m} \mathrm{~s}^{-1}$ or 4 degrees longitude per day westward. This is the speed of the zonal mean zonal flow near the center latitude, and thus the critical latitude of the most unstable mode (and emergent Kelvin cat's eyes) is very close to the center latitude of the model domain $\left(18^{\circ}\right.$ latitude). 

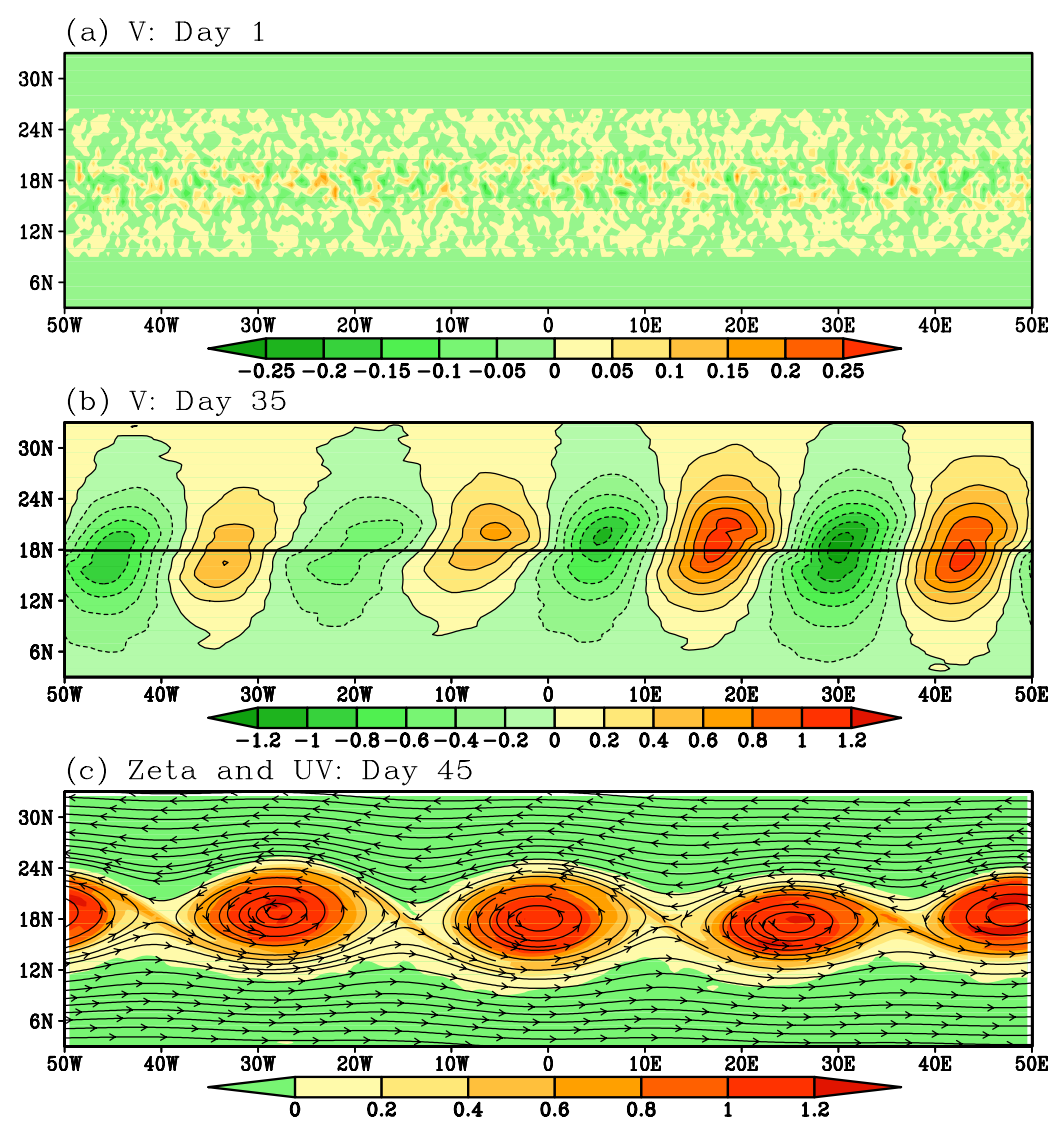

Fig. 2. Meridional wind disturbance (unit: $\mathrm{m} \mathrm{s}^{-1}$ ) at sea surface from the idealized dry model simulation (see text for details) at (a) Day 1 and (b) Day 35, and (c) relative vorticity (unit: $10^{-5} \mathrm{~s}^{-1}$ ) and streamlines at Day 45 in the co-moving frame of reference that moves at the zonal phase speed of the waves $\left(-5 \mathrm{~m} \mathrm{~s}^{-1}\right)$.

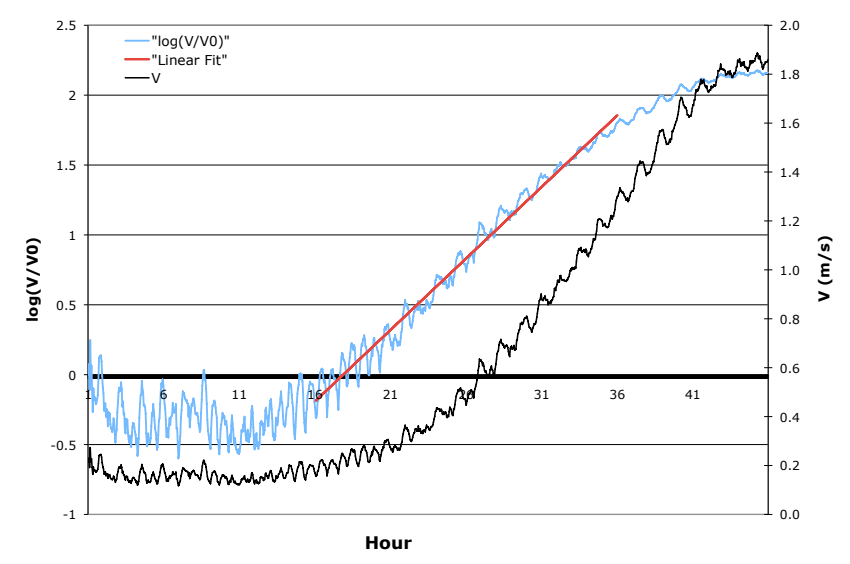

Fig. 3. Time series of the surface maximum meridional wind (black; $y$-axis on the right) and its natural logarithm (blue; $y$-axis on the left) from the dry WRF simulation. The red straight line is a linear fit on a log-linear scale from Day 16 to Day 35, implying an efolding time of approximately 9.8 days.
As a check on whether the domain is large enough to select the most unstable mode for this zonal flow, we extended the domain zonally to $120^{\circ}$ longitude, and the dominant wave response did not change. This suggests that the wave with $2800 \mathrm{~km}$ wavelength (or $25^{\circ}$ longitude) is indeed the most unstable wave for this basic flow, subject to the integer multiple constraint: viz., wavenumber 4 or 5 in the 100 or 120 deg domains, respectively. The upper tropospheric disturbance is wavenumber 3 in the 100 deg domain and evidently decoupled from the lower tropospheric wave.

It might be argued that having to wait 45 days for the emergence of a nascent gyre-pouch with maximum cyclonic relative vorticity of only $1.5 \times 10^{-5} \mathrm{~s}^{-1}$ is far too long to be of practical relevance for tropical cyclogenesis in the Western Atlantic or other basins such as the Western Pacific for which the zonal mean flow might serve as a reasonable approximation in certain flow regimes. However, these dry lifecycle experiments usefully illustrate how easily a random wind perturbation can project onto the intrinsic cat's eye flow structures that are supported by these types of cyclonic shear flows. 
For the remainder of this paper we adopt the same methodology as KT81 and investigate the disturbance-to-storm transformation process by employing a small but finite amplitude initial perturbation having approximately the same structural characteristics (horizontal wavelength and vertical decay scale) of the dominant unstable (dry) wave pattern found above, without the meridional phase tilt, and at approximately three times the saturation amplitude for relative vorticity $\left(\sim 4.5 \times 10^{-5} \mathrm{~s}^{-1}\right)$. The corresponding moist experiments conducted herein therefore adopts the "clumpiness" of the cyclonic gyre-pouches from the dry experiment, but magnifies their value by approximately a factor of three. Although the genesis sequence presented below has been verified to be essentially similar to its small-amplitude counterpart experiment, this scaling renders our set up virtually identical to that of KT81 and gives an arguably more realistic transformational time scale from a precursor disturbance.

Following KT81, the zonal domain is set to $25^{\circ}$ longitude and the initial wave disturbance is defined from the following expressions for the zonal and meridional wind perturbation in Cartesian coordinates:

$u^{\prime}$
$=\left\{\begin{array}{l}-9 \frac{y}{L_{y}^{2}} A(\sigma) \cos \left(\frac{2 \pi x}{L_{x}}\right) \exp \left(-\frac{9}{2}\left(\frac{y}{L_{y}}\right)^{2}\right) \\ \text { if }\left|\frac{y}{L_{y}}\right|<1 \\ 0 \text { otherwise }\end{array}\right.$

$=\left\{\begin{array}{l}\frac{2 \pi}{L_{x}} A(\sigma) \sin \left(\frac{2 \pi x}{L_{x}}\right)\left[\exp \left(-\frac{9}{2}\left(\frac{y}{L_{y}}\right)^{2}\right)-\exp \left(-\frac{9}{2}\right)\right] \\ \text { if }\left|\frac{y}{L_{y}}\right|<1 \\ 0 \text { otherwise }\end{array}\right.$

where $L_{y}=952 \mathrm{~km}\left(8.5^{\circ}\right), L_{x}=2800 \mathrm{~km}\left(25^{\circ}\right)$ and $A(\sigma)$ defines the vertical profile of the disturbance

$A(\sigma)=\frac{L_{x}}{2 \pi} v_{0}\left\{\begin{array}{l}1, \text { for } \sigma \geq \sigma_{0} \\ \cos ^{2}\left(\frac{\pi}{2} \frac{\sigma-\sigma_{0}}{L_{\sigma}}\right), \text { for } \sigma_{0}>\sigma>\sigma_{0}-L_{\sigma} \\ 0, \text { for } \sigma \leq \sigma_{0}-L_{\sigma}\end{array}\right.$

where $v_{0}=5 \mathrm{~m} \mathrm{~s}^{-1}, L_{\sigma}=0.35$, and $\sigma_{0}=0.7$. Figure $4 \mathrm{a}-\mathrm{c}$ shows plan views of the zonal, meridional and relative vorticity fields at the lowest sigma level over one wavelength. From Fig. 4d, e we see that the initial meridional and zonal wind disturbance is largely confined below the $500 \mathrm{hPa}$ level and has maximum amplitude at the surface. As noted by KT81 (p 1634), "The decrease of wave amplitude with height implies, under the assumption of a balance condition, lack of a cold core structure. In this respect the present disturbance resembles certain waves observed in the Pacific ... more closely than those in the Atlantic."
Following KT81, the surface pressure, three-dimensional temperature and geopotential fields are derived from the initial wind field by solving the reverse balance equation in Cartesian-sigma coordinates (see Appendix A for details). Rather than adjust these initial fields to a steady-state frictional boundary layer (as in KT81) we let the WRF model perform this adjustment. The boundary layer adjustment occurs on a time scale on the order of $1 / \sqrt{(} f \zeta_{\text {abs }}$ ) (cf. Eliassen and Lystad, 1977), where $\zeta_{\mathrm{abs}}$ is the absolute vertical vorticity of the zonal mean flow $(f-\partial U / \partial y)$. For $f$ corresponding to $18^{\circ} \mathrm{N}$, the adjustment time is less than $39 \mathrm{~h}$.

\subsubsection{Inclusion of moisture}

To include moisture, we specify a vertical profile of relative humidity $(r)$, and then deduce the air temperature $(T)$ and mixing ratio $(q)$ from virtual temperature $\left(T_{\mathrm{V}}\right)$ by iteration upon using the Magnus formulae:

$\left\{\begin{array}{l}T=T_{\mathrm{v}} /\left(1+\varepsilon_{1} q\right) \\ \mathrm{e}_{s}=\left\{\begin{array}{l}1000 \varepsilon_{1} \exp \left[\varepsilon_{2}\left(T-\varepsilon_{0}\right) /\left(T-\varepsilon_{3}\right)\right] \text { if } T>\varepsilon_{0} \\ 1000 \varepsilon_{1} \exp [21.87(T-273.16) /(T-7.66)]\end{array} \text { otherwise }\right. \\ q_{s}=\varepsilon_{4} \mathrm{e}_{s} /\left(p-\mathrm{e}_{s}\right) \\ q=q_{s} r\end{array}\right.$

where $q_{s}$ is the saturation mixing ratio, $p$ is total pressure and $\mathrm{e}_{s}$ is the saturation vapor pressure in $\mathrm{Pa}$. The constants are defined as follows: $\varepsilon_{0}=273.15 \mathrm{~K}, \varepsilon_{1}=0.6112, \varepsilon_{2}=17.67$, $\varepsilon_{3}=29.65 \mathrm{~K}$, and $\varepsilon_{4}=0.62198$.

Following KT81, we specify a horizontally homogeneous relative humidity field on constant pressure surfaces. This is a reasonable approximation to the observed moisture field over the West Atlantic. The vertical cross-section of mixing ratio is shown in Fig. 4f. Because of the cyclonic (anticyclonic) wave-like disturbance that is added to the zonal basic state flow, a corresponding low (high) surface pressure is present near the domain center. From Eq. (6b), this implies a slightly enhanced (reduced) mixing ratio near the center of the domain on constant pressure surfaces (Fig. 4f).

\subsubsection{Wave/pouch tracking method}

In our previous diagnoses using coarse-resolution global model products (DMW09; Wang et al., 2009), Hovmöller diagrams of meridional wind perturbations or moisture field were used for wave tracking. So as to obtain consistent results from the high-resolution simulations, the propagation speed of the wave/pouch is determined by tracking of the pouch centroid. The pouch centroid $(\bar{x}, \bar{y})$ is defined as

$\left\{\begin{array}{l}\bar{x}=\frac{1}{n} \sum_{\text {pouch }} x_{i} \\ \bar{y}=\frac{1}{n} \sum_{\text {pouch }} y_{i}\end{array}\right.$

where $i$ denotes the grid points within the pouch and $n$ is the total number of grid points within the pouch. We define 
(a) U

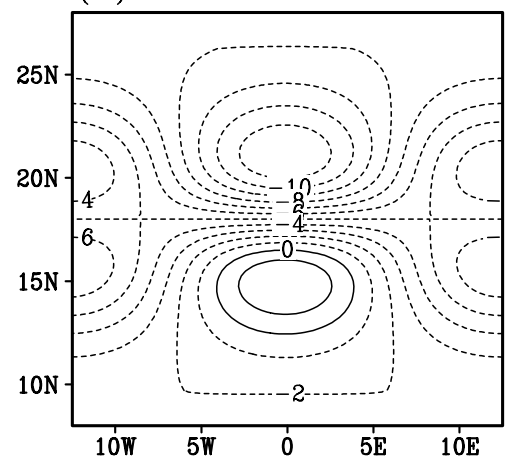

(b) $\mathrm{V}$

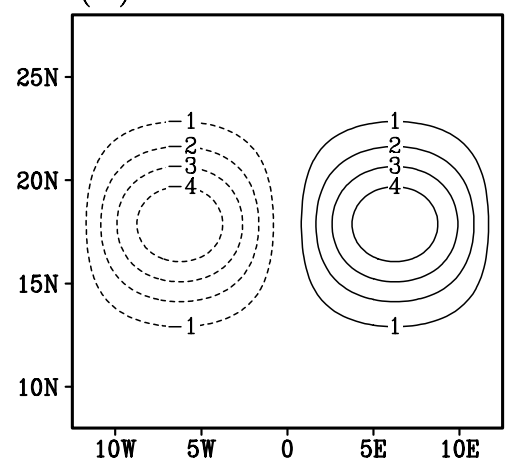

(c) zeta

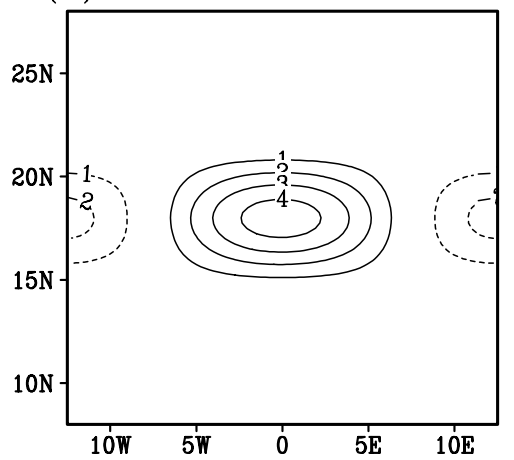

(d) $\mathrm{U}^{\prime}(\mathrm{OE})$

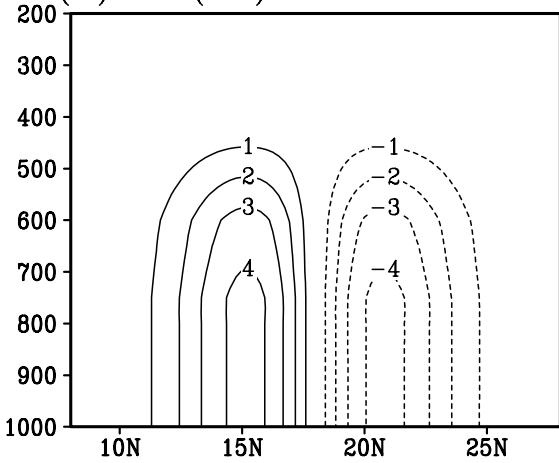

(e) $\mathrm{V}^{\prime}(6.25 \mathrm{~W})$

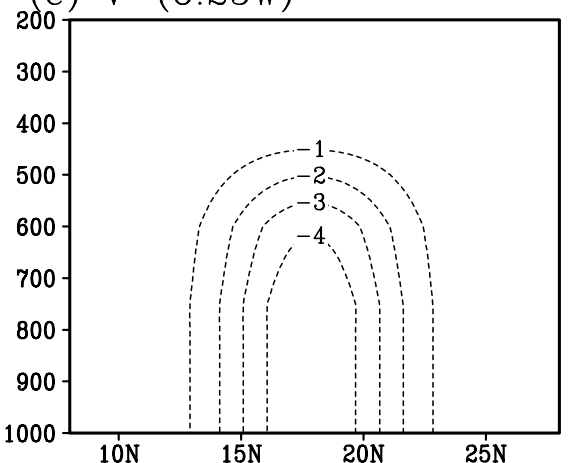

(f) $Q(\mathrm{OE})$

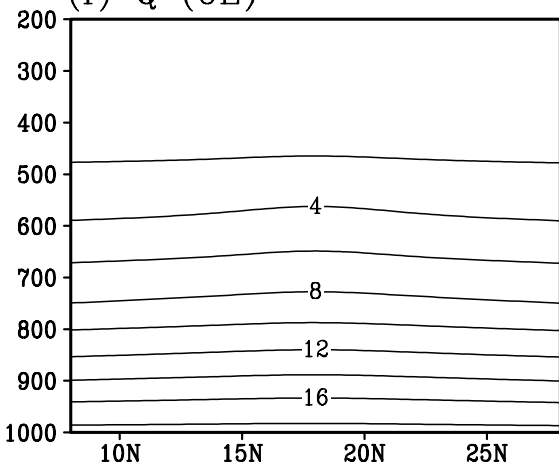

Fig. 4. The left panels show the initial zonal wind $\left(\mathbf{a}\right.$; unit: $\left.\mathrm{ms}^{-1}\right)$, meridional wind $\left(\mathbf{b}, \mathrm{ms}^{-1}\right)$ and relative vorticity $\left(\mathbf{c}\right.$; unit: $\left.10^{-5} \mathrm{~s}^{-1}\right)$ at the surface, and the right panels show the vertical cross section of the initial zonal wind perturbations (d), meridional wind perturbations (e) and mixing ratio $\mathrm{Q}$ (f; unit: $\mathrm{g} / \mathrm{kg}$ ). Perturbations are defined as the difference between the total field and the basic state.

the pouch by the closed stream function contours in the comoving frame. For our present purposes, we determine the propagation of the pouch in a two step fashion. The first step determines the closed stream function contours in the resting frame of reference (as shown in Fig. 5, the so-called "streamfunction gyre") and calculates the corresponding zonal propagation of its area-centroid. The second step uses this first estimate of the propagation speed to determine the closed stream function in the the co-moving frame (also shown in Fig. 5) and obtain an updated propagation speed of the corresponding centroid. In practice, the second step gives only a small correction (of a few percent) to the first guess for the propagation speed and for this reason we have neglected the second step for the calculation of the propagation speed in the majority of the experiments presented here.

The advantage of the centroid method is that since the centroid is defined based on the spatial average within the closed streamfunction gyre in the resting frame (or pouch in the comoving frame), high-frequency wind fluctuations exert little influence on the track of the centroid. As a result, the estimated zonal propagation speed (e.g., Fig. 6) is slowly varying with time and rather robust. Unless otherwise noted, the 


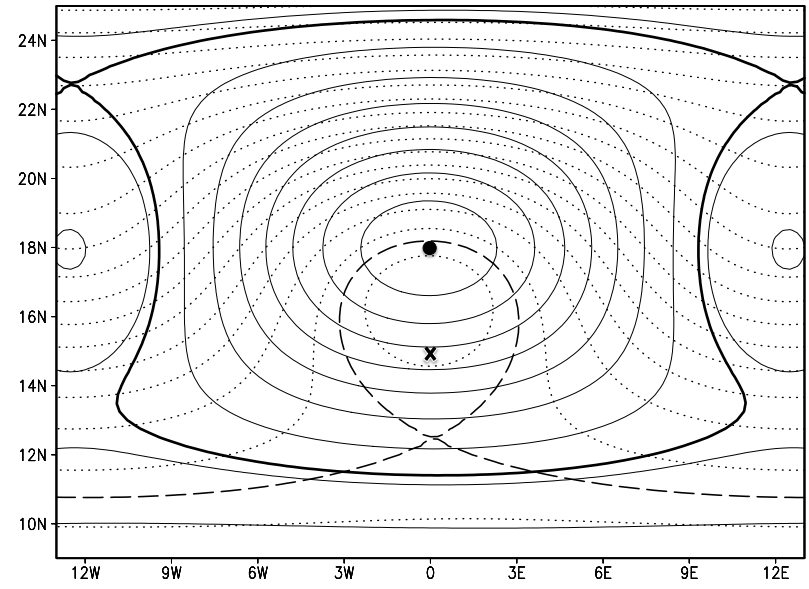

Fig. 5. Stream function in the resting frame of reference (dotted contours) and in the co-moving frame of reference (solid contours). The thick solid line is the bounding contour of the wave pouch in the co-moving frame of reference, and the thick dashed line delineates the closed circulation in the resting frame (stream function gyre). The circle and the cross indicate the pouch center and the mass centroid of resting stream function gyre, respectively.

(a) Longitude of the Centroid
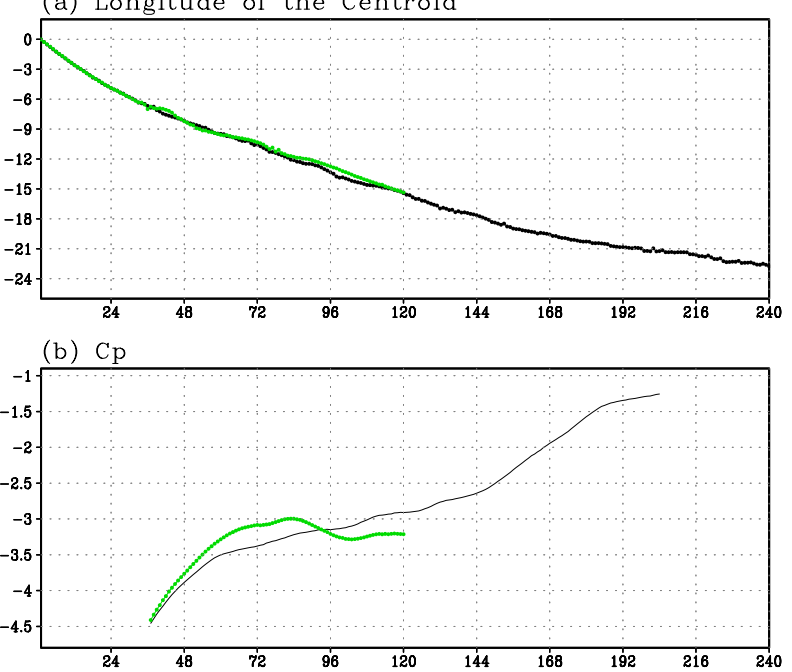

Fig. 6. (a) The longitude of the mass centroid and (b) the propagation speed $(\mathrm{Cp})$ of the gyre-pouch based on its vorticity centroid as described in the text. The black curves represent the coarseresolution simulation, and the green curves represent the warm-rain experiment. The abscissa is time (h). The ordinate is longitude in (a) and propagation speed $\left(\mathrm{m} \mathrm{s}^{-1}\right)$ in (b).

time-varying propagation speed as obtained above is used to translate the frame of reference, and all pertinent diagnoses related to the vorticity dynamics and thermodynamics of the pouch region are carried out in the co-moving frame and are centered on the pouch centroid.
Comparison of pouch structure in the co-moving frame as initialized in the moist runs (Fig. 5) with that produced by the dry run (Fig. 2) indicate that both have a region of closed cyclonic circulation centered at $18^{\circ} \mathrm{N}$, with minor differences in kinematic structure near the periphery of the pouch. They share a common feature having a central "core" region of positive Okubo-Weiss parameter (following the sign convention of DMW09) implying a region favorable for vortex aggregation and shape preservation of the meso-beta vorticity aggregate or "proto-storm."

\section{Numerical experiments and supporting interpretations}

\subsection{KT81 revisited: coarse-resolution $(28 \mathrm{~km})$ simulation}

In the control run, the relative humidity is specified as in KT81, so that the planetary boundary layer is uniformly moist with relative humidity values of approximately $85 \%$, consistent with observations over the West Atlantic during the hurricane season.

Figure 7 shows time series for maximum wind speed at $850 \mathrm{hPa}$, surface pressure and $850 \mathrm{hPa}$ relative vorticity. Three time series are shown for each of the three variables, corresponding to an increasing level of complexity in the representation of moist processes. The black curves denote the coarse resolution simulation using the Betts-Miller-Janjic cumulus parameterization scheme; the green curves depict the high-resolution "warm-rain" simulation, and the blue curves depict the high-resolution simulation with full ice microphysics (WRF Single-Moment 6-class scheme; Hong and Lim, 2006). The coarse resolution experiment will be discussed here; discussion of the latter two simulations that explicitly represent cloud processes will be deferred to the upcoming sections.

The horizontal grid spacing in the coarse resolution simulation is $28 \mathrm{~km}$ (or $0.25^{\circ}$ ) in contrast with the somewhat larger grid spacing of $0.625^{\circ}$ in KT81. The maximum horizontal wind speed at $850 \mathrm{hPa}$ attains minimal hurricane strength at approximately $144 \mathrm{~h}$ and decreases in intensity after $180 \mathrm{~h}$. The minimum surface pressure drops slightly below $990 \mathrm{hPa}$ soon after $144 \mathrm{~h}$. The maximum $850 \mathrm{hPa}$ relative vorticity is $1.5 \times 10^{-3} \mathrm{~s}^{-1}$ and is attained a little earlier at $128 \mathrm{~h}$. While the time series is broadly useful for summarizing the time evolution of certain fields, it does not reveal the cyclogenesis pathway. The central question to be addressed here is what is the transformational process between the initial wave-like disturbance and the emergent tropical storm?

Figure 8 shows the evolution of stream function and relative vorticity in the frame of reference that moves at the same speed as the easterly wave. At early times the initially broad-scale cyclonic vortex within the wave's surface low stimulates the formation of multiple mesoscale 

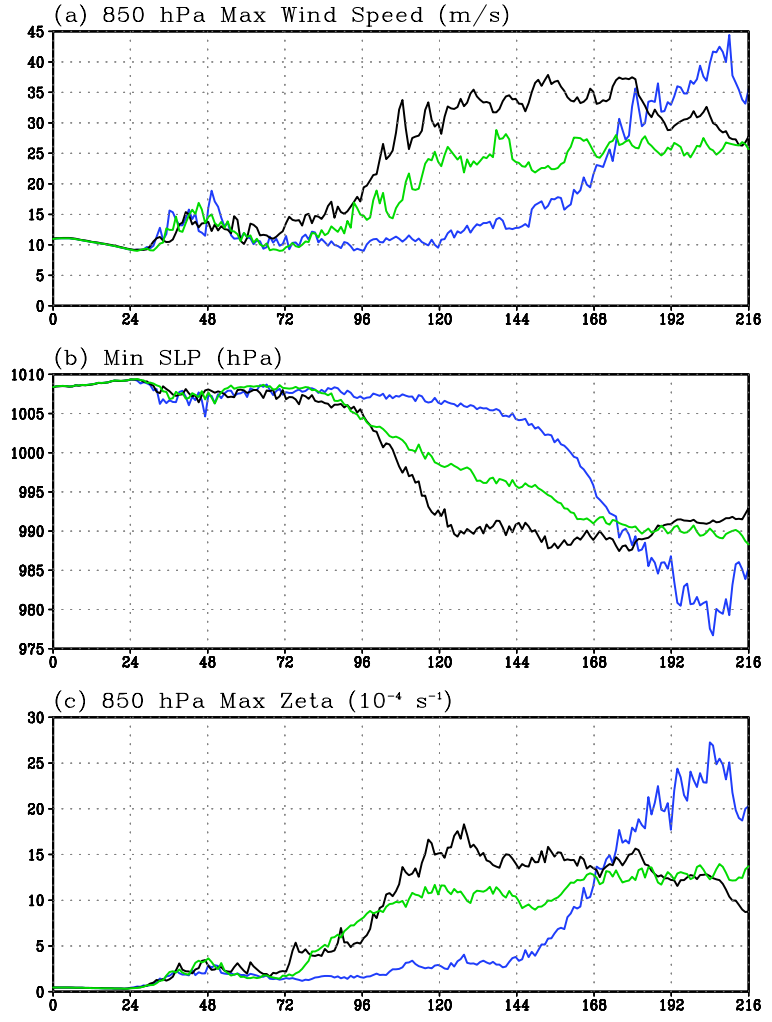

Fig. 7. Time evolution of $850 \mathrm{hPa}$ maximum wind speed (a), surface pressure (b), and $850 \mathrm{hPa}$ maximum relative vorticity (unit: $10^{-4} \mathrm{~s}^{-1}$ ) (c). The black curve is for the single-grid, coarseresolution simulation, the green curve is the high-resolution, nested simulation with warm rain microphysics, and the blue curve is the high-resolution, nested simulation with ice microphysics. The abscissa is time $(\mathrm{h})$.

vortices that subsequently intensify in the lower troposphere by vortex tube stretching in regions of deep (parameterized) moist convection. Generally, these vortices outlive the convection that creates them and tend to merge with neighboring remnant vortices or become axisymmetrized ("scavenged") by younger and more intense convective vortices. These simulated vortical structures generally have the strongest winds near the top of, or within, the (inflow) boundary layer. From a vorticity budget perspective (not shown here; see upcoming high resolution analysis for more) the mesoscale vortex enhancement process is such that the intensification of the near-surface horizontal winds leads that of the middle levels, and by this definition is always from the bottom up (Montgomery and Enagonio, 1998; Montgomery et al., 2006; Tory et al., 2006a, b and Tory et al., 2007). Furthermore, the mesoscale vorticity enhancement occurs most persistently near the intersection of the wave trough and the critical latitude for the moist waves (purple curve). This intersection point exhibits a striking "attractor-like" quality in the ensuing development process.
A close up view of the evolving stream function (solid curves) and relative vertical vorticity (color) during the time interval $78 \mathrm{~h}-111 \mathrm{~h}$ is highlighted in Fig. 8. The evolution from $84 \mathrm{~h}$ to $108 \mathrm{~h}$ shows an example of merging mesoscale vortices. In this experiment, deep convective activity and low-level vorticity enhancement continue more or less unabated near the central latitude, resulting in a strong tropical storm strength vortex with maximum winds at $850 \mathrm{hPa}$ of approximately $35 \mathrm{~m} \mathrm{~s}^{-1}$ after $144 \mathrm{~h}$ integration time (Fig. 7a). The moral of the story suggested by this experiment is that there is an upscale, "bottom up" development that occurs within the wave's surface low, very near the critical latitude of the parent (dry) wave disturbance. In this example the critical latitude is located coincidentally where the vertical shear of the zonal flow is minimal (cf. Montgomery et al., 2006) and the meridional gradient of the basic state vorticity changes sign.

Figure 9a shows a snapshot of the Okubo-Weiss parameter (defined in DMW09 as OW $=\zeta^{2}-S_{1}^{2}-S_{2}^{2}$ ) at $96 \mathrm{~h}$, a time at which a tropical storm-strength vortex has emerged from within the parent wave's pouch. As shown by McWilliams (1984), OW $>0$ indicates that the flow is vorticity dominant, whereas $\mathrm{OW}<0$ indicates that the flow is strain rate-dominant. Regions with large positive OW tend to be immune from the filamentary enstrophy cascade. In the initial stage of pouch evolution (not shown), the weak mesoscale vortices are characterized by weak, positive OW embedded in a local environment which is strain rate-dominant $(\mathrm{OW}<0)$. As the mesoscale vortices become stronger, they exhibit a structure similar to a tropical storm: at this time there are three distinct VHTs forming a curved band near the center of the pouch and these are marked by regions of strong positive OW (red/yellow/light-green). This positive OW region is surrounded by a strain-rate dominant region (purple/dark-blue).

Figure $9 \mathrm{~b}-\mathrm{d}$ shows a snapshot of horizontal divergence and equivalent potential temperature $\left(\theta_{e}\right)$ at the $850 \mathrm{hPa}$ level, and saturation fraction (column relative humidity between surface and $500 \mathrm{hPa}$ ) at $96 \mathrm{~h}$. Whereas the $850 \mathrm{hPa}$ divergence field is noisy, as is to be expected in a strongly convective environment, strongly convergent regions (purple) possessing magnitude on the order of $10^{-4} \mathrm{~s}^{-1}$ are clearly evident near the center of the pouch. These maxima are approximately collocated with the intense cyclonic mesoscale vortices (cf. Fig. 8) and OW maxima at this time. The corresponding field $\theta_{e}$ (Fig. 9c) has maximum values that are generally collocated with convergence maxima. Additionally, the $\theta_{e}$ field outside this region possesses a spiral band structure in which bands of elevated $\theta_{e}$ spiral cyclonically inward, lending support to the terminology "feeder bands" (Guinn and Schubert, 1993). Finally, the saturation fraction (SF, Fig. 9d) indicates a nearly saturated column of moist tropical air in the center of the pouch with maxima again collocated with strong convergence or updrafts. The "feeder bands" are more evident in the SF field, and they 
Zeta (AOKT Run, 850 hPa)
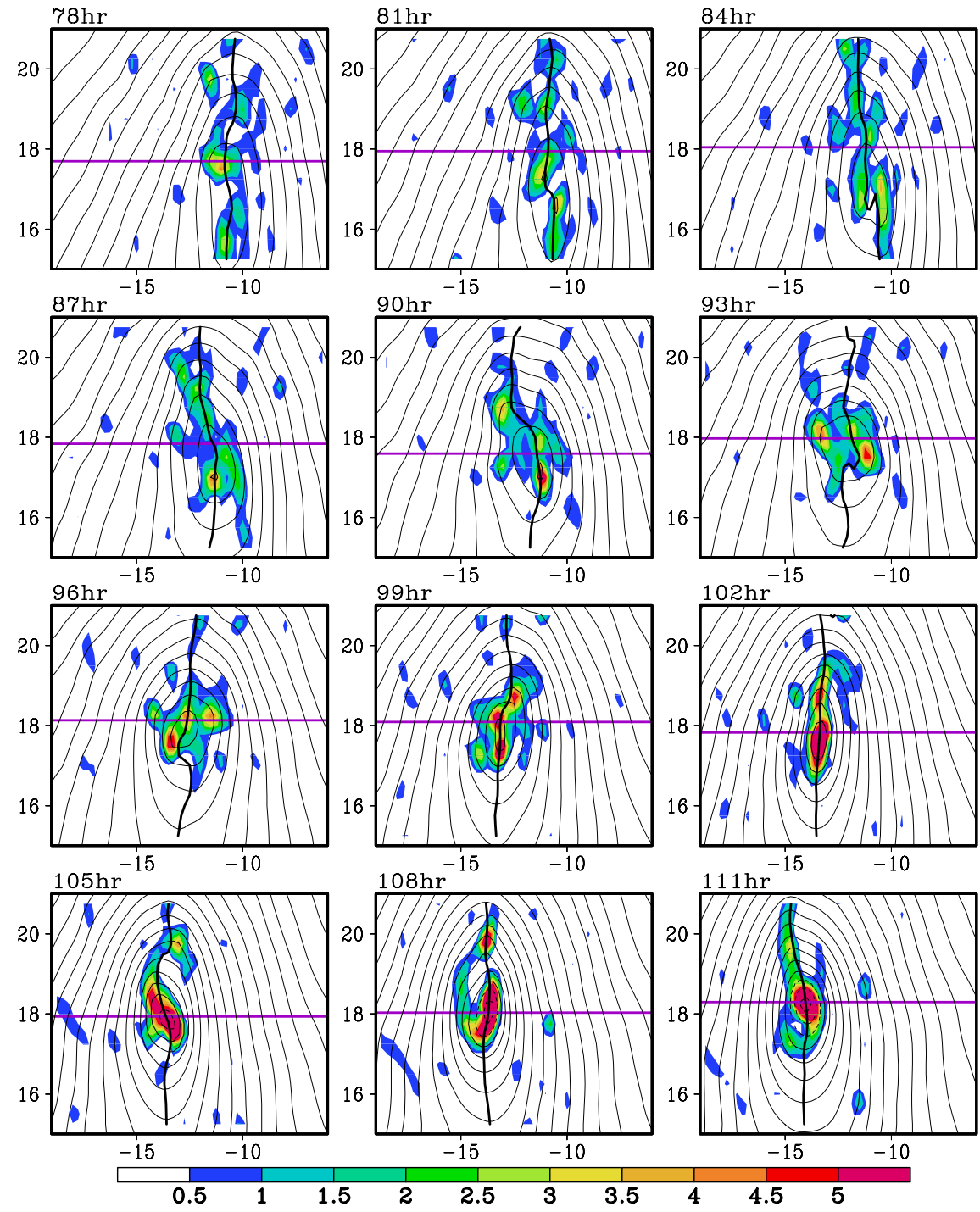

Fig. 8. Evolution of stream function and relative vorticity (color) in the coarse-resolution (control) run from hour 78 to hour 111 in the co-moving frame of reference that moves at the zonal speed of the parent easterly wave. The unit of the vorticity is $10^{-4} \mathrm{~s}^{-1}$. The black curve is the wave trough axis and the purple line indicates the (linear) critical latitude (see text for details). The ordinate is latitude and the abscissa is longitude.

are associated with parameterized cumulus convection (not shown). These features are consistent with KT81 (their Figs. 5-8 and Fig. 22), who found multiple convective systems within the surface low.

In rotating moist convection problems that commence with a relatively dry middle troposphere, one expects to see negative $\theta_{e}$ anomalies emerge within and just above the boundary layer in association with convective downdrafts that import (dry) low $\theta_{e}$ air into the boundary layer (e.g., Rotunno and Emanuel, 1987). However, with the BettsMiller-Janiic cumulus parameterization scheme, downdrafts are not explicitly active so convective downdrafts are nonexistent, and consequently, the $\theta_{e}$ deficits in the boundary layer are considerably weaker than their counterpart simulations with explicit downdrafts (e.g., M06). (The lack of explicit downdrafts in the KT81 study has been a lingering concern by some tropical cyclone researchers. This restriction is removed in the upcoming high-resolution simulations in Sects. 3.2 and 3.3.)

It proves useful to introduce a new diagnostic that highlights the evolution of the maximum relative vorticity along each latitude near and within the wave's cyclonic critical 
Hour 96, $850 \mathrm{hPa}$
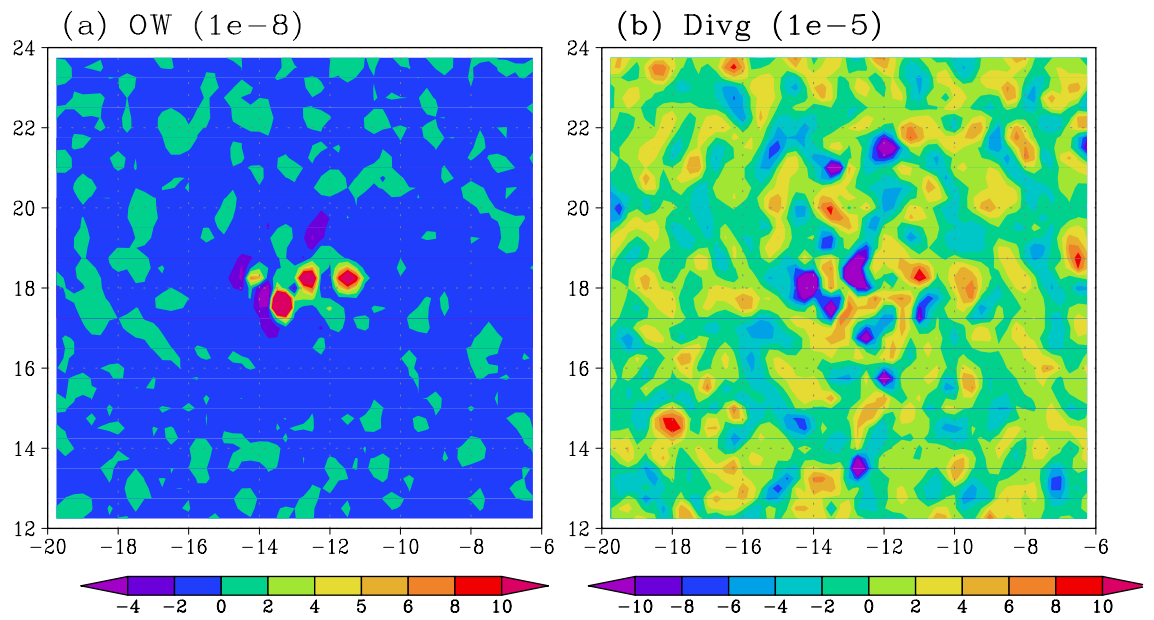

(c) Theta_e (K)
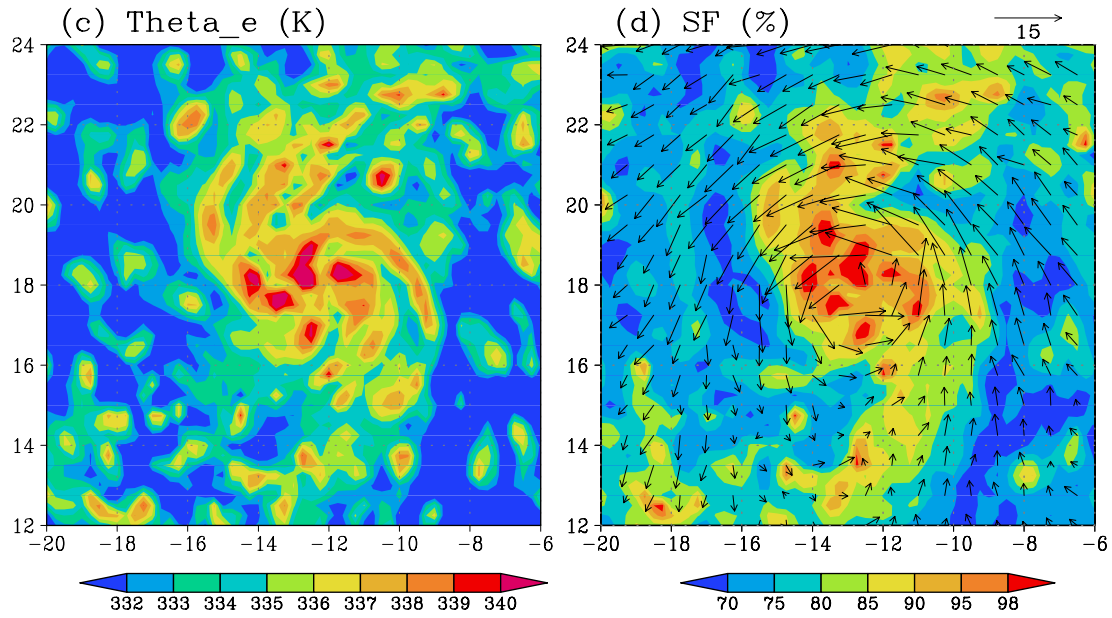

Fig. 9. OW (a, unit: $10^{-8} \mathrm{~s}^{-2}$ ), divergence (b, unit: $\left.10^{-5} \mathrm{~s}^{-1}\right), \theta_{e}(\mathrm{~K})$ (c), saturation fraction from the surface to $500 \mathrm{mb}$ (shading in d), and wind field (vectors in $\mathrm{d}$ ). The ordinate is latitude and the abscissa is longitude.

layer. Figure 10a shows cyclonic vorticity with values larger than $1.5 \times 10^{-4} \mathrm{~s}^{-1}$ only. A concentrated and intense mesoscale vortex appears around $96 \mathrm{~h}$ (Day 4) near the original critical latitude of the parent wave $\left(18^{\circ} \mathrm{N}\right)$. After this time the vorticity intensifies primarily in a highly localized spatial region near the critical latitude, and the emergent vortex starts a slow northwestward drift away from the original critical latitude. The chaotic swarming of vorticity maxima about the critical latitude (prior to $96 \mathrm{~h}$ ) followed by the highly focused vorticity concentration is a striking feature. The multiple vortex activity exhibited prior to the attractor-type behavior is somewhat reminiscent of T. Fujita's infamous "suction vortices" first hypothesized from retrospective Tornado damage surveys (Fujita, Mystery of Severe Storms, 1991). The multiple vortices evident in this figure are obviously considerably larger in horizontal extent than tornado suction vortices, but these vortices are nonetheless smaller in horizontal scale than the tropical storm vortex and represent a class of analogous sub-vortex scale structures. This type of diagram will hereafter be referred to as a "Fujita diagram".

Figure 10b shows the Fujita diagram of the analyzed saturation fraction (column relative humidity from the surface to $500 \mathrm{mb}$ ). The emergent vortex, which develops into a tropical storm, forms in a region with high saturation fraction. Before storm formation the horizontal scale of the region of

\footnotetext{
${ }^{1}$ The link with Fujita's work is in reference to the multiple vortex nature of the genesis process within the pouch rather than the mechanism responsible for the multiple vortex structures. In the tornado, the origin of the suction vortices is thought to be due to a shear instability associated with a two-celled vortex. Prior to the generation of a tropical storm, the small-scale cyclonic vorticity anomalies originate from convective instability and the associated vortex-tube stretching of like-sign vorticity of the local environment in the lower troposphere.
} 

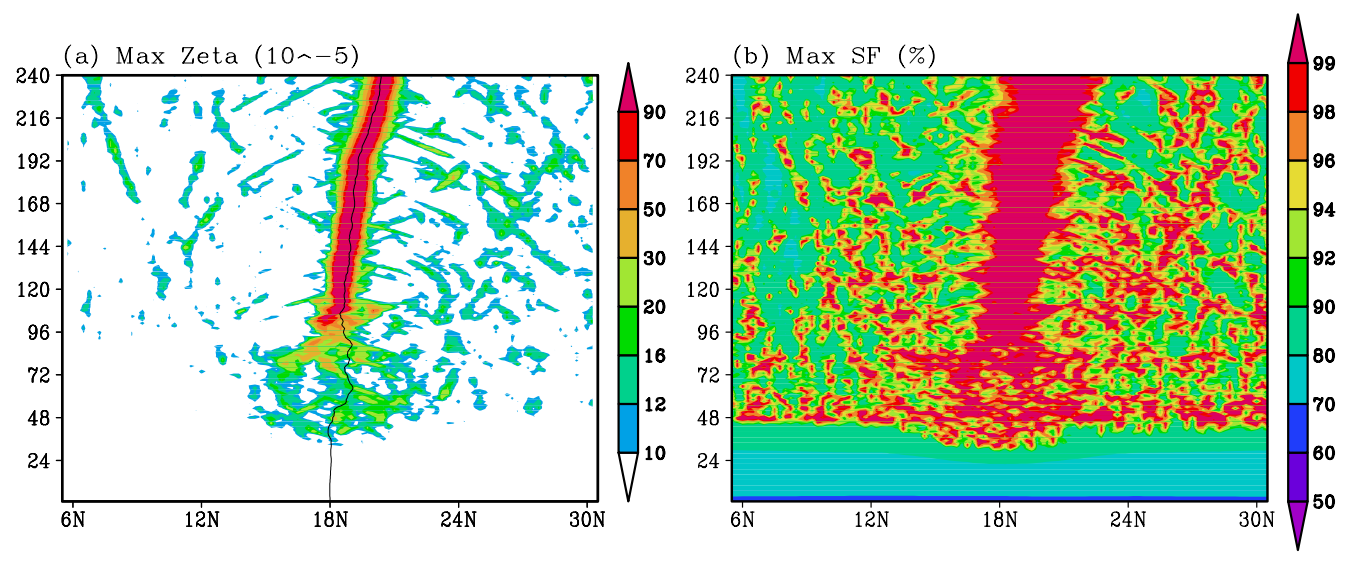

Fig. 10. "Fujita Diagram:" (a) depicts the time evolution of the maximum relative vorticity along each latitude. The $x$-axis indicates the latitude of the vorticity maximum and the $y$-axis is time (from Day 0 to Day 10); (b) depicts the time evolution of the maximum saturation fraction from surface to $500 \mathrm{hPa}$. The ordinate is time (h).

(a) Initial UV and Zeta $(\mathrm{X} 10 \wedge-5)$

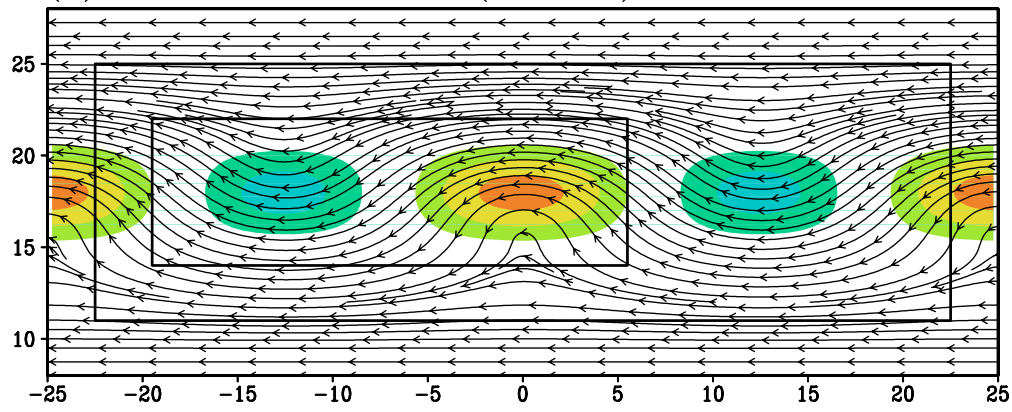

(b) Hour $120(\mathrm{X} 10 \wedge-4)$

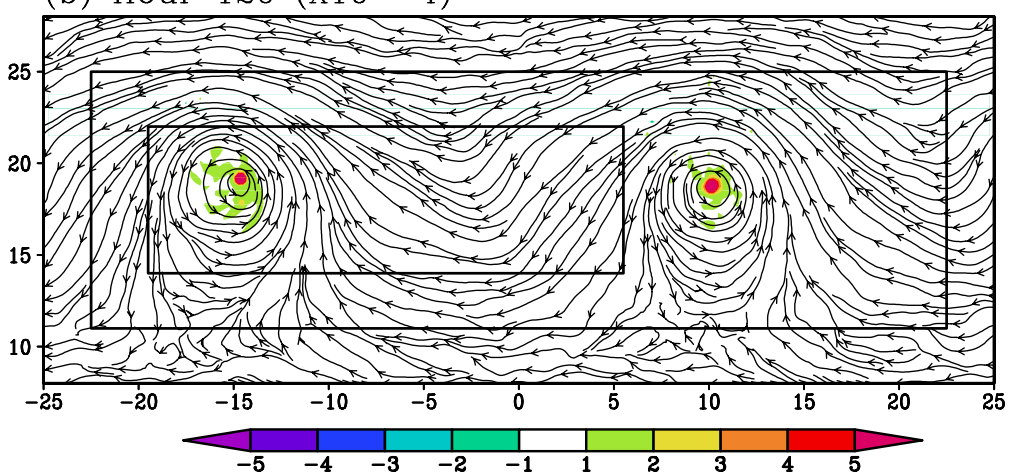

Fig. 11. The 3-grid nested simulation: Relative vorticity and the corresponding (Earth-relative) streamlines at the initial time (a) and after $120 \mathrm{~h}$ integration (b). The two rectangles represent the two inner grids. The ordinate is latitude and the abscissa is longitude.

high saturation fraction is comparable to that of the region of large cyclonic vorticity. After consolidation to a single master vortex there is a broader "funnel" of saturation fraction.

\subsection{KT81 high-resolution simulation $(3.1 \mathrm{~km})$ with "warm rain" microphysics}

As a simple way of testing both the robustness of the KT81 results and the new interpretation presented herein, we move progressively to a nested grid numerical simulation of the same experiment conducted above. The experimental set up is highlighted in Fig. 11a. Three grid domains are indicated 
by the solid black rectangles. The coarse horizontal grid spacing is $28 \mathrm{~km}$ (the same as before); the intermediate horizontal grid spacing is approximately $9.3 \mathrm{~km}$; while the fine grid spacing is approximately $3.1 \mathrm{~km}$. The horizontal extent of the outer grid is increased to two wavelengths ( $50^{\circ}$ longitude), and the innermost grid contains only one full wavelength. For the time period chosen, the cyclonic trough region of the eastern wave spends the majority of its time within the intermediate resolution $(9.3 \mathrm{~km})$ grid domain where the Betts-Miller-Janiic cumulus parameterization is operative, and the cyclonic trough region of the western wave spends all of its time in the high resolution $(3.1 \mathrm{~km})$ grid with explicit moist convection operating therein (Fig. 11b). In this experiment, the WRF warm rain scheme is used, and the effects of ice microphysics are neglected.

An advantage of this experiment is that one can assess the relative importance of explicit moist processes (warm rain and ensuing precipitation driven downdraft phenomenology) and parameterized moist processes within the same numerical experiment. For example, from the vorticity field shown in Fig. 11b, a concentrated tropical storm-like vortex has formed by $120 \mathrm{~h}$ on both the intermediate and fine grids. (The fine and intermediate grid fields have been interpolated up to the coarsest grid, $28 \mathrm{~km}$.) Although the storms are located at approximately the same latitude, a consolidated tropical-storm vortex looks more well developed in the intermediate grid. This is confirmed from the time series of $850 \mathrm{hPa}$ horizontal wind speed, minimum sea level pressure and $850 \mathrm{hPa}$ relative vertical vorticity (not shown). The maximum horizontal wind speed and sea-level pressure deficit predicted on the fine grid are reduced relative to the coarse grid simulation. The primary reason for the delayed development in the warm rain scheme lies with the occurrence of precipitation-induced downdrafts which import relatively low $\theta_{e}$ into the boundary layer, thereby interrupting the moistening and destabilization of the lower troposphere by sea-to-air fluxes of water vapor (e.g., Montgomery et al., 2010). For the remaining discussion we present pertinent highlights of the warm rain simulation in as much as they support or refute the coarse resolution test and the proposed marsupial paradigm.

\subsubsection{Wave and VHT evolution from high- resolution simulation}

As is evident in the translated stream function and precipitation rate patterns in Fig. 12, the initially broad-scale cyclonic vortex within the wave's surface low stimulates the formation of multiple precipitation events associated with the vortical hot plumes represented by the high-resolution grid (not shown). The horizontal purple line in each figure denotes the (linear) critical latitude defined by equating the zonal mean velocity to the zonal wave propagation speed (Fig. 6). At early times $(\sim 72 \mathrm{~h})$ precipitation is widely distributed within the wave's pouch. At later times, however, precipitation in- creases and becomes more and more concentrated near the center of the pouch. At long times ( $\sim 120 \mathrm{~h}$; not shown) maximum precipitation is coincident with the pouch center and the intersection of the wave trough axis and the (linear) wave critical latitude.

An example of the merger of the mesoscale vortices or their vortical remnants is shown in Fig. 13. At $82 \mathrm{~h}$, there are two prominent cyclonic vortices in the northeast quadrant of the pouch. They move westward with the pouch. Meanwhile, the weaker vortex (B) rotates cyclonically around the stronger vortex (A), and it merges with the stronger vortex by $84.4 \mathrm{~h}$. The resultant vortex has a stronger intensity and larger size than the individual vortices, and it is associated also with high surface $\theta_{e}$ as shown in the right column in Fig. 13. This dominant vortex moves to the center of the pouch by $87 \mathrm{~h}$ (not shown). As confirmed by the Fujita diagram shown in Fig. 14, the vortex development has transitioned from a swarm of approximately independent VHTs and their vortical remnants to a single master vorticity monolith immersed in a nearly saturated tropospheric column (Fig. 14b). This attractor-like evolution within the wave pouch noted previously persists here.

Based on the experiments presented in this paper, and in a companion study examining the cyclogenesis of preHurricane Felix (2007) (Wang et al., 2010a, b), this attractorlike behavior appears to be a ubiquitous property of the simulated wave-to-storm transition process. The focal point is the nominal center of the Kelvin cat's eye of the finite-amplitude tropical easterly wave (or the center of the wave pouch). From the perspective of linear wave dynamics, the preference for persistent convection near this focal point was already anticipated theoretically, as well as observationally using the NASA/TRMM precipitation products, in DMW09. Recall that along the critical latitude the intrinsic frequency vanishes and fluid parcels are able to reside the longest there. During this time, parcels experience persistent moistening from the underlying ocean and latent heating associated with deep cumulus convection. Because these fluid parcels are immersed in a cyclonic-rich vorticity environment, the latent heating will result in the stretching of the cyclonic vortex tubes passing though these parcels in the lower troposphere. Barring unfavorable conditions, these two properties virtually guarantee a rapid intensification of the local cyclonic vorticity near the center of the cat's eye flow. The "sweet spot" of the cat's eye (defined here as the intersection between trough axis and critical latitude in the lower troposphere) is thus the preferred location for tropical cyclogenesis and the findings herein support this novel prediction of the marsupial paradigm.

\subsubsection{Mesoscale evolution}

A mesoscale view of the wave-to-storm transition process is obtained next by calculating horizontal averages of key variables over a spatially limited portion of the wave pouch 


\section{Precip and 850 hPa Psi}
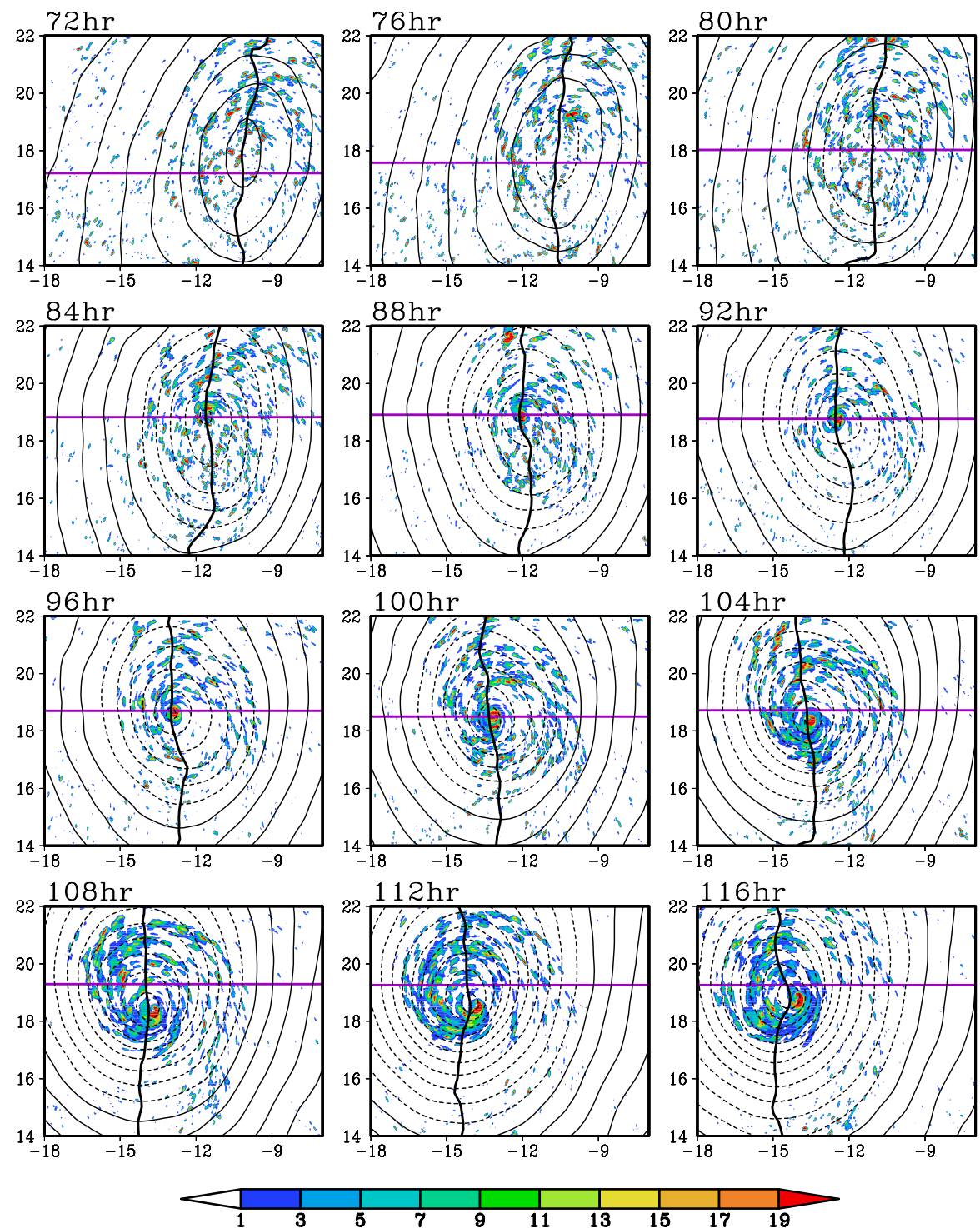

Fig. 12. Evolution of translated stream function and precipitation (shading; unit: $\mathrm{mm} / \mathrm{h}$ ) from the inner grid ( $3 \mathrm{~km}$ resolution) simulation from Hour 72 to Hour 116. The zonal straight line indicates the critical latitude and the meridional black curve represents the trough axis. The ordinate is latitude and the abscissa is longitude.

containing the sweet spot, and displaying the results in a time-height format. Figure 16 presents the results from such an analysis for a $2^{\circ}$ latitude by $2^{\circ}$ longitude box centered on the translating sweet spot. Shown are vertical distributions of area-averaged relative vorticity, horizontal divergence, relative humidity and $\theta_{e}$ fields. Figure 15 highlights certain aspects of this area-average analysis in a time series format.

Before examining the details of Fig. 15, it is helpful to note that the fine grid is switched on at $t=46 \mathrm{~h}$ and as a result there is some adjustment of the fields on the fine grid until approximately $t=66 \mathrm{~h}$. Before the fine grid is switched on, the area- averaged cyclonic vorticity increases as in the coarse resolution simulation. Once the fine grid is switched on, however, the deep convective processes trigger convective downdrafts (not shown), and for an interval of approximately $20 \mathrm{~h}$, the area-averaged surface vorticity and $\theta_{e}$ weaken until the boundary layer has moistened sufficiently (cf. Fig. 4 of Rotunno and Emanuel, 1987).

As shown in Fig. 15, after $t=66 \mathrm{~h}$, the near-surface vorticity ( $900 \mathrm{hPa}$ Zeta) intensifies until $t=90 \mathrm{~h}$ when the intensification is interrupted briefly; amplification is then renewed with a somewhat larger rate. Also evident after $t=66 \mathrm{~h}$ is 

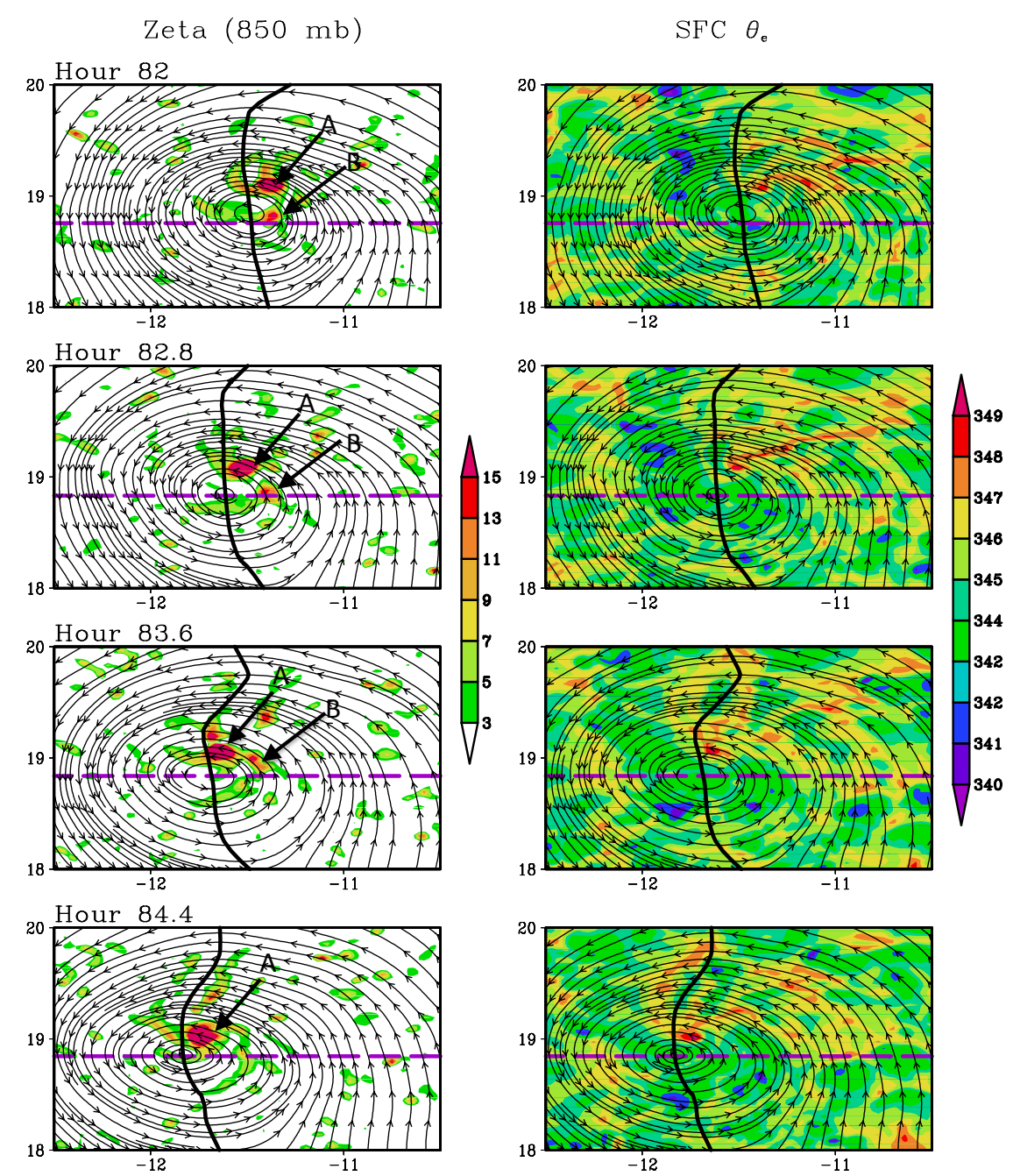

Fig. 13. Evolution of VHTs from Hour 82 to Hour 84.4 ( $3 \mathrm{~km}$ mesh). The left panels are $850 \mathrm{hPa}$ relative vorticity (unit: $\left.10^{-4} \mathrm{~s}^{-1}\right)$, the right panels are the surface $\theta_{e}(\mathrm{~K})$, and both are superimposed on the $850 \mathrm{hPa}$ translated streamlines. The black curve is the wave trough axis, and the purple line represents the wave critical latitude. The two vortices discussed in the text are indicated by black arrows and letters (A and B) in the left panels. The ordinate is latitude and the abscissa is longitude.

that the amplification of near-surface vorticity leads the amplification of mid-level vorticity ( $500 \mathrm{hPaZeta})$. As in the coarse grid simulation, the simulated vortex development is thus from the bottom up.

Once the fine grid is turned on the boundary layer RH always exceeds $85 \%$ (see Fig. 16c); during the grid transition period the RH quickly increases to near saturation values (>95\%), remaining as such thereafter. Since the near-surface $\mathrm{RH}$ is nearly saturated after $t=60 \mathrm{~h}$, the near-surface $\mathrm{RH}$ is not useful for predicting the genesis time. It has been suggested recently that the increase in midlevel humidity may be a "trigger" for tropical cyclogenesis. The proposal is that the increase of midlevel RH generally precedes the amplification of surface vorticity and as result this might be a crucial observable for forecasting the timing of cyclogenesis (Nolan,
2007). It is clear from Fig. 15, however, that in the context of the tropical wave-to-cyclone transition problem as posed by KT81 and by us, the increase of mid-level relative humidity $(500 \mathrm{hPa} \mathrm{RH})$ does not precede the amplification of nearsurface vorticity $(900 \mathrm{hPaZeta})$; rather, it accompanies the spin up of near-surface vorticity, and both follow the commencement of the low-level convergence ( $900 \mathrm{hPa}$ Divg). The evolution of relative humidity at $400 \mathrm{hPa}$ or $600 \mathrm{hPa}$ is similar to that at $500 \mathrm{hPa}$.

The time-height evolution of divergence, relative vorticity, relative humidity and $\theta_{e}$ is shown in Fig. 16. The time-height plot of horizontal divergence shown in Fig. 16b depicts a fairly deep low- to mid-level column of divergence during the coarse-to-fine grid transition period $(48 \mathrm{~h}<t<66 \mathrm{~h})$; this is associated with the precipitation-induced downdrafts and 

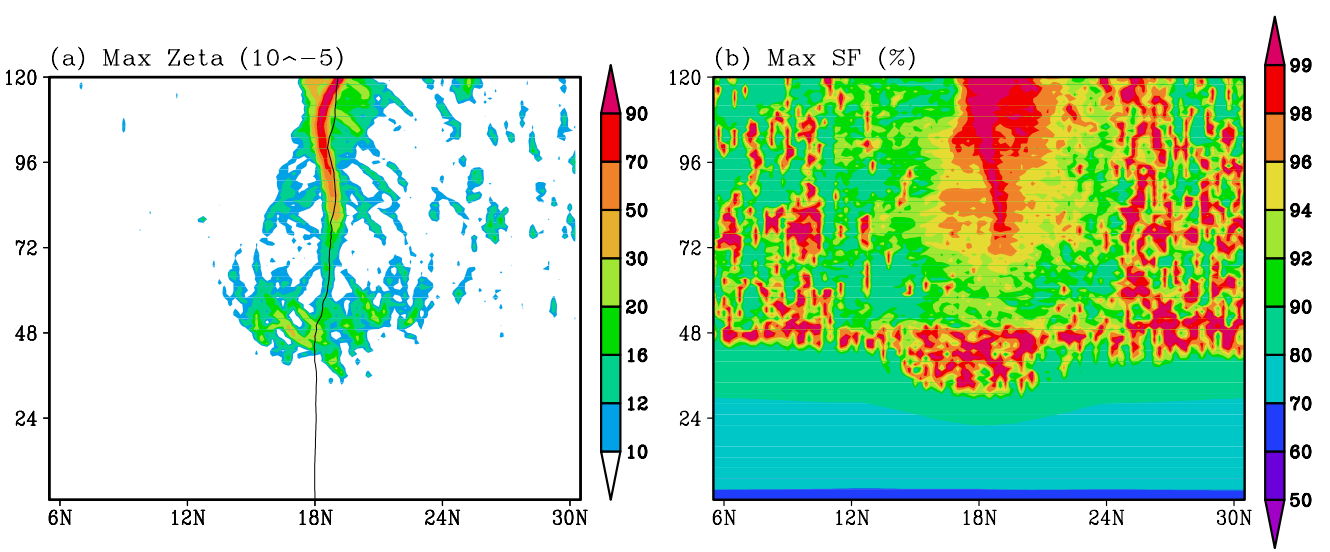

Fig. 14. Fujita diagram at high resolution: Time evolution of the $850 \mathrm{hPa}$ maximum relative vorticity (left; unit: $10^{-5} \mathrm{~s}-1$; derived from the 3-km horizontal grid output) and saturation fraction (SF) along each latitude. The $x$-axis indicates the latitude of the vorticity or SF maximum, and the $y$-axis is time (from Hour 25 to Hour 120). The thin curve in the left panel indicates the latitude where the zonal mean vertical shear between $200 \mathrm{hPa}$ and $850 \mathrm{hPa}$ is zero.

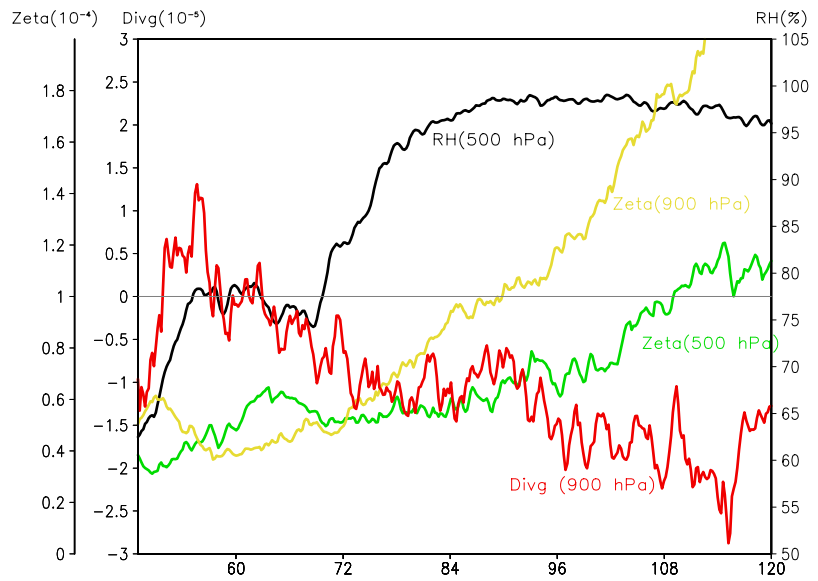

Fig. 15. Time evolution of $500 \mathrm{hPa}$ relative humidity (unit: \%), $900 \mathrm{hPa}$ divergence (unit: $10^{-5} \mathrm{~s}^{-1}$ ), $500 \mathrm{hPa}$ and $900 \mathrm{hPa}$ relative vorticity (unit: $10^{-4} \mathrm{~s}^{-1}$ ) averaged in a $2^{\circ} \times 2^{\circ}$ box following the moving pouch center from Hour 50 to Hour 120 .

the attendant low-level divergence patterns that accompany these downdrafts. Even during this transition time, however, there is a clear surface convergence signature. Soon after $t=66 \mathrm{~h}$, a moderately deep convergent column spanning the low- to mid-troposphere replaces the previous divergence pattern. The convergence within this layer intensifies slowly; the top of convergent layer also descends with time until the top is below the $850 \mathrm{hPa}$ level for $t>120 \mathrm{~h}$. Subsequently, all of the convergence is confined to a relatively thin boundary layer. (The upcoming section will suggest that this latter property is likely due to the absence of the ice microphysics in this simulation.) The divergence field at $t=66 \mathrm{~h}$ is confined to above $450 \mathrm{hPa}$, but with time the level of zero divergence gradually descends to approximately $850 \mathrm{hPa}$ at $120 \mathrm{~h}$.
The time-height plot $\theta_{e}$ in Fig. 16 depicts a gradually decreasing surface $\theta_{e}$ during the early phase of the genesis process $(48 \mathrm{~h}<t<90 \mathrm{~h})$ until a time at which rapid intensification of the emergent vorticity monolith is well underway. This is unlike the widely accepted view that the increase of the localaverage vorticity should be accompanied by an increase in the local-average $\theta_{e}$ near the ocean surface, as expected in the familiar WISHE mechanism of tropical cyclone intensification articulated by Emanuel et al. (1994). This finding is supported by recent observational work (Molinari et al., 2004) and theoretical/modeling work (Montgomery et al., 2006; Tory et al., 2006b) indicating that tropical cyclogenesis is not a WISHE process. These studies suggested that the amplification process during the genesis stage is dominated by deep and locally buoyant convective vortical plumes that are able to extract sufficient moisture from the boundary layer without significant augmentation of the sensible and latent heat fluxes associated with an increase in the surface wind speed on the vortex scale.

\subsubsection{Mesoscale vorticity budget}

Similar to the procedure expounded in Haynes and McIntyre (1987) and the methodology outlined in Weisman and Davis (1998), Cram et al. (2002) and Davis and Trier (2002), we use the flux form of the vorticity equation in pressure coordinates and calculate area-averages of the vertical vorticity tendency equation in flux form:

$\frac{\partial \eta}{\partial t}=-\frac{\partial}{\partial x}\left(u \eta+\omega \frac{\partial v}{\partial p}-G\right)-\frac{\partial}{\partial y}\left(v \eta-\omega \frac{\partial u}{\partial p}+F\right)$

where $\eta$ is the absolute vorticity defined as $\eta=f+\partial v / \partial x-$ $\partial u / \partial y$, and $F$ and $G$ represent the zonal and meridional components, respectively, of the effects of additional forces, such as friction and subgrid scale processes. Here $p$ denotes 

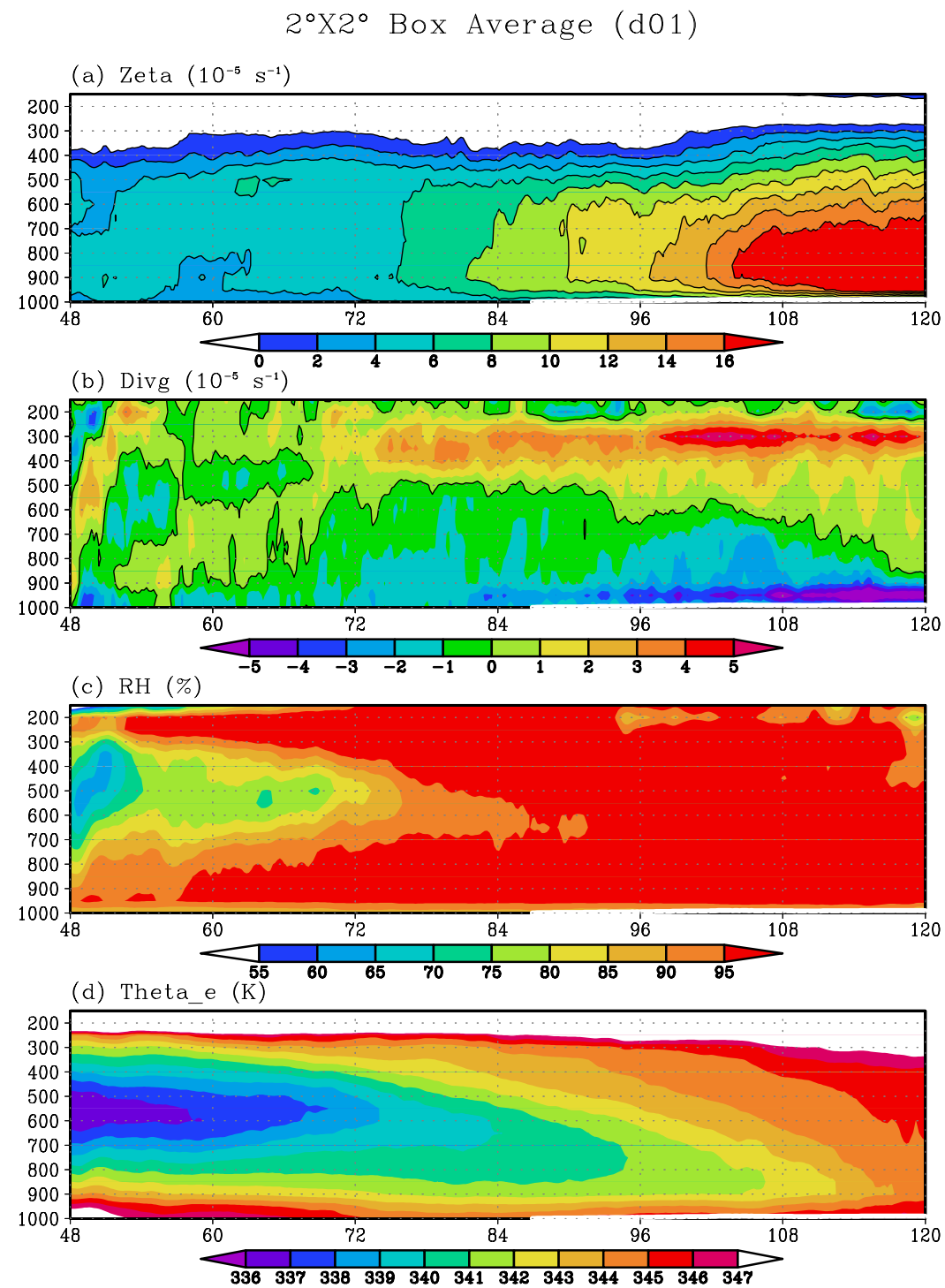

Fig. 16. The vertical distribution of the $2^{\circ} \times 2^{\circ}$ box averages of (a) relative vorticity (unit: $10^{-5} \mathrm{~s}^{-1}$ ), (b) divergence (unit: $\left.10^{-5} \mathrm{~s}^{-1}\right),(\mathbf{c})$ relative humidity (unit: \%) and (d) $\theta_{e}$ (unit: K) following the moving pouch center (defined by the intersection of the local critical surface and the trough axis) from Hour 48 to Hour 120. The ordinate is pressure (hPa) and the abscissa is time (hour). Values below the surface are masked out between hours 87 to 120 .

pressure and it is understood that horizontal derivatives are taken on pressure surfaces. Since the sub-grid scale processes are typically more than an order of magnitude smaller than the friction terms associated with the surface boundary layer, we may estimate the frictional contribution to vorticity tendency by residual using the calculated tendency and advective terms. Consistent with the methodology used to create Fig. 16, the pertinent velocities used in Eq. (8) are defined relative to the nearly constant translation speed of the sweet spot defined in the previous section. To reduce the high-frequency fluctuations, a one-hour running time average is applied to each budget term.
In addition to avoiding the inherent problem of measuring the small difference between large terms that can occur when using the material form of the vorticity equation (Haynes and McIntyre, 1987), the strength of this method lies in the fact that, through Gauss's theorem, the tendency of the circulation for any enclosed area fixed in space can be written in terms of the line integral of the flux component normal to the boundary of the area (Davis and Trier, 2002):

$$
\frac{\partial C}{\partial t}=\iint_{A} \frac{\partial \eta}{\partial t} d A=-\oint\left(\vec{V} \eta-\omega \widehat{k} \times \frac{\partial \vec{V}}{\partial p}+\vec{R}\right) \cdot \widehat{n} d l
$$


where $C$ is the absolute circulation, $A$ represents the area encompassed by the closed loop, $\vec{V}$ the horizontal velocity, and $\vec{R}=(-G, F)$. We will refer to the first term under the integral as the horizontal convergence of absolute vorticity (horizontal flux term). A comparison between the flux and the material forms of the vorticity equation (Holton, 1992) reveals that the horizontal flux term in Eq. (9) is related to the horizontal advection term plus the stretching term in the material form of the vorticity equation. The second term is an amalgamation of the vertical advection of vertical vorticity and the tilting of horizontal vorticity into vertical vorticity and will be hereafter denoted simply as the tilting-like (or non-advective) term (see Tory and Montgomery, 2008 for clarification of this terminology). For comparison, Davis and Trier (2002) refer to these terms as stretching and tilting, respectively.

The budget terms are illustrated in Fig. 17. They are calculated by averaging the vorticity flux divergences in a $2^{\circ} \times 2^{\circ}$ box as done in Fig. 16, which by Stokes' theorem is equivalent to the line integral of the corresponding flux through the lateral boundary. Figure 17a shows that the area-averaged vorticity tendency during the period between $66 \mathrm{~h}$ and $80 \mathrm{~h}$ is generally positive between the low- to mid-troposphere and the boundary layer, which is consistent with the weak convergence from the low- to mid-troposphere as shown in Fig. 16b. After $t=80 \mathrm{~h}$, however, Fig. 17b shows that the horizontal convergence of vertical vorticity becomes more focused in the lower troposphere and it is eventually largest in a shallow boundary layer $(t>100 \mathrm{~h}$ ) (the top of which we associate nominally with the depth of the strong convergence layer). Since the tilting-like term shown in Fig. 17c (which includes the resolved convective momentum transport) makes a second-order contribution to the calculated vorticity budget, and since the friction term (as calculated by residual in Fig. 17d) is maximized within this layer and is always negative there, the only process that can account for the spin up of cyclonic near-surface vorticity at these later times is horizontal convergence of vertical vorticity within the boundary layer. This is borne out clearly in Fig. 17b.

The spin up of near-surface vorticity after $t=100 \mathrm{~h}$ merits some discussion in light of recent studies arguing that friction, when confined to the sub-cloud layer, should always cause a spin down of the near-surface flow (e.g., Raymond et al., 1998; Raymond and Sessions, 2007; Marni et al., 2008). While it is correct that friction generally retards fluid motions, it will soon become clear that such reasoning is overly simplistic in vortical flows possessing VHTs whose aggregate effect is to establish a near-surface convergence of absolute angular momentum (Hendricks et al., 2004; Montgomery et al., 2006). For the present numerical simulation we have calculated the complete vorticity budget. In order to account for the spin up of the emergent tropical storm it was not necessary to assume a "boundary layer depth of $4 \mathrm{~km}$ " (cf. Raymond et al., 1998).
Recent work by Smith et al. (2009) and Bui et al. (2009) have demonstrated that there are generally two spin up mechanisms operating in tropical cyclones. The first is associated with a diabatically-forced convergence of absolute angular momentum above the frictional boundary layer. The second is via a diabatically-forced but frictionally modified inertial inflow that is maximized within the boundary layer. In the latter case, the frictional boundary layer can generate a positive spin up effect provided the convergence of absolute angular momentum acts fast enough to overcome the corresponding transfer of angular momentum into the underlying ocean. This is essentially what happens post genesis $(\mathrm{t}>100 \mathrm{~h})$ in Figs. 16 and 17.

The moral of the story is that the spin up of near-surface vorticity in the experiments conducted here results primarily from the near-surface convergence associated with the diabatically forced inflow from the buoyant VHTs. Moreover, during the post-genesis/intensification phase, the leadingorder surface spin up mechanism is from convergence within the frictional boundary layer, which is again driven primarily by the buoyant VHTs near the pouch center. This assessment is consistent with the findings reported in our companion papers (Wang et al., 2010a, b) for the real-case simulation of the genesis of pre-Hurricane Felix (2007).

\subsection{KT81 high resolution simulation $(3.1 \mathrm{~km})$ with ice microphysics}

In this section we take up the issue of the modification of the above genesis sequence due to ice microphysical processes. The motivation for this section is manifold. One hypothesis, as extreme as it may seem, is that the results and interpretation will change fundamentally when ice microphysical processes are included. To be sure, the use of rain-only physics provides an incomplete picture of the mesoscale processes occurring within the pouch; in particular, it does not address the hypothesized relative role of convective and stratiform convective processes in the genesis sequence (DMW09). We employ the same nested grid configuration used in Sect. 3.2 and the same boundary layer and cumulus parameterization on the coarsest mesh $(28 \mathrm{~km})$. The only difference is that Kessler warm-rain microphysics scheme is replaced by the WRF single-moment, 6-class microphysics scheme (Hong et al., 2006) which includes ice, snow and graupel processes.

The time series of the high resolution simulation with ice microphysics is summarized in Fig. 7. As in the warm rain experiment, the fine grid is activated at $t=44 \mathrm{~h}$. For all three metrics shown, the ensuing development is significantly delayed relative to the warm rain experiment. It is not until approximately $t=180 \mathrm{~h}$ that the ice microphysics simulation surpasses the intensity of the warm rain experiment, presumably because of the stronger downdrafts associated with ice processes (Srivastava, 1987). 


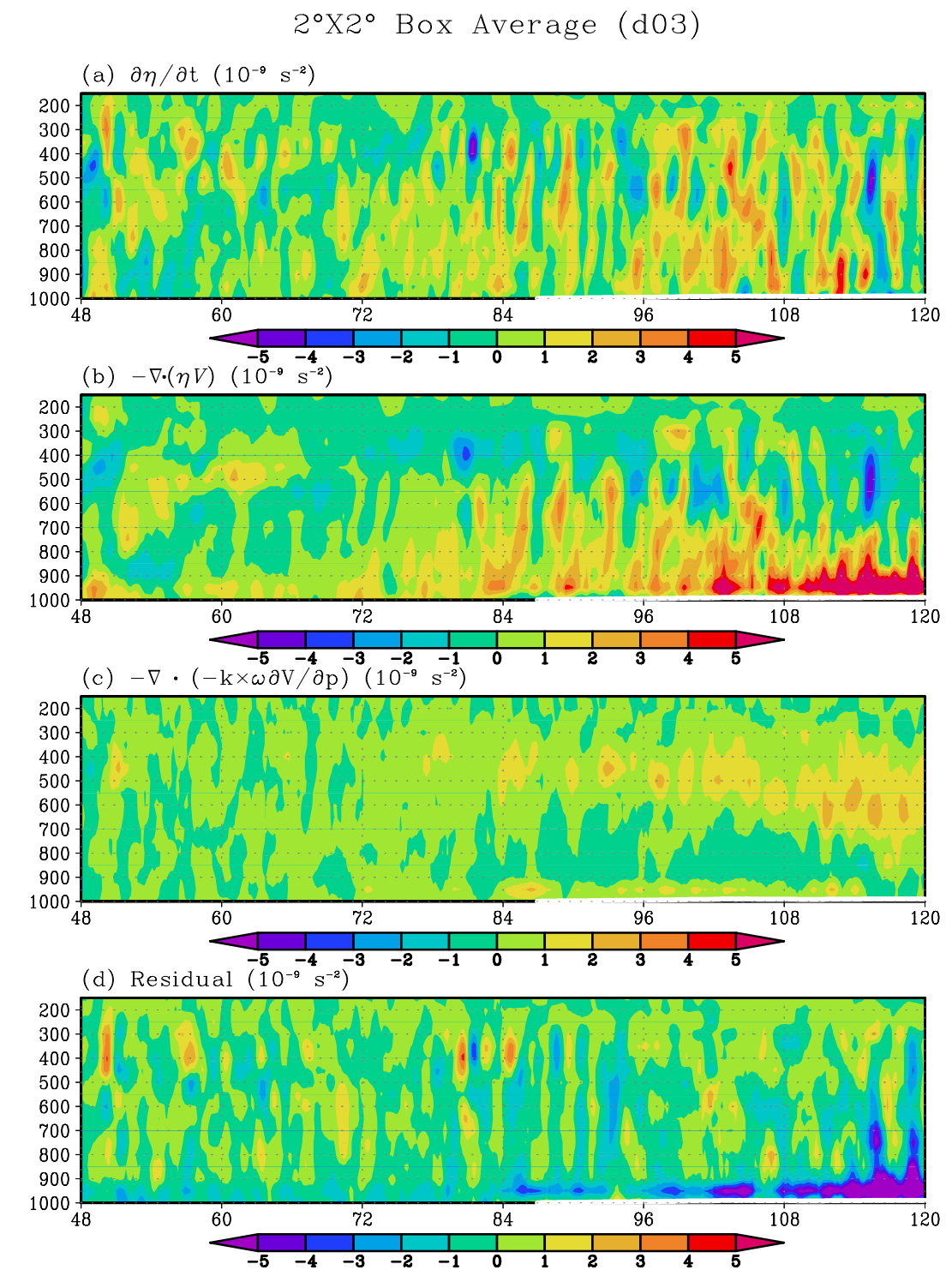

Fig. 17. Vorticity budget (averaged over a 2-degree $\times 2$-degree box following the pouch center) from Hour 48 to Hour 120 . All fields are derived from the inner most grid (d03) simulation and a one-hour running mean was applied to reduce high-frequency fluctuations. The vorticity tendency unit is $10^{-9} \mathrm{~s}^{-2}$. The ordinate is pressure $(\mathrm{hPa})$ and the abscissa is time $(\mathrm{h})$.

\subsubsection{Mesoscale evolution}

The mesoscale view of the wave-to-storm transition process is presented in Fig. 18, following the same methodology used to construct Fig. 16. Shown are vertical distributions of areaaveraged relative vorticity, horizontal divergence, relative humidity and $\theta_{e}$ fields.

Beginning around $t=66 \mathrm{~h}$, the near-surface vorticity slowly intensifies. This is accompanied by an amplification of the low- to middle troposphere vorticity field. As in the warm rain experiment, we see a deep convergent layer that becomes progressively shallower on the whole with time as the system vortex intensifies. Consequently, the region of mean divergence deepens with time, but retains its maximum in the upper troposphere as expected. The convergence is maximized in the boundary layer and becomes pronounced when the system vortex begins to intensify rapidly $(t>144 \mathrm{~h}$, Fig. 7). A time series of the near-surface and $500 \mathrm{hPa}$ vorticity (not shown) shows that the amplification of surface vorticity leads the amplification of the $500 \mathrm{hPa}$ vorticity. The development process is thus again from the bottom up. 

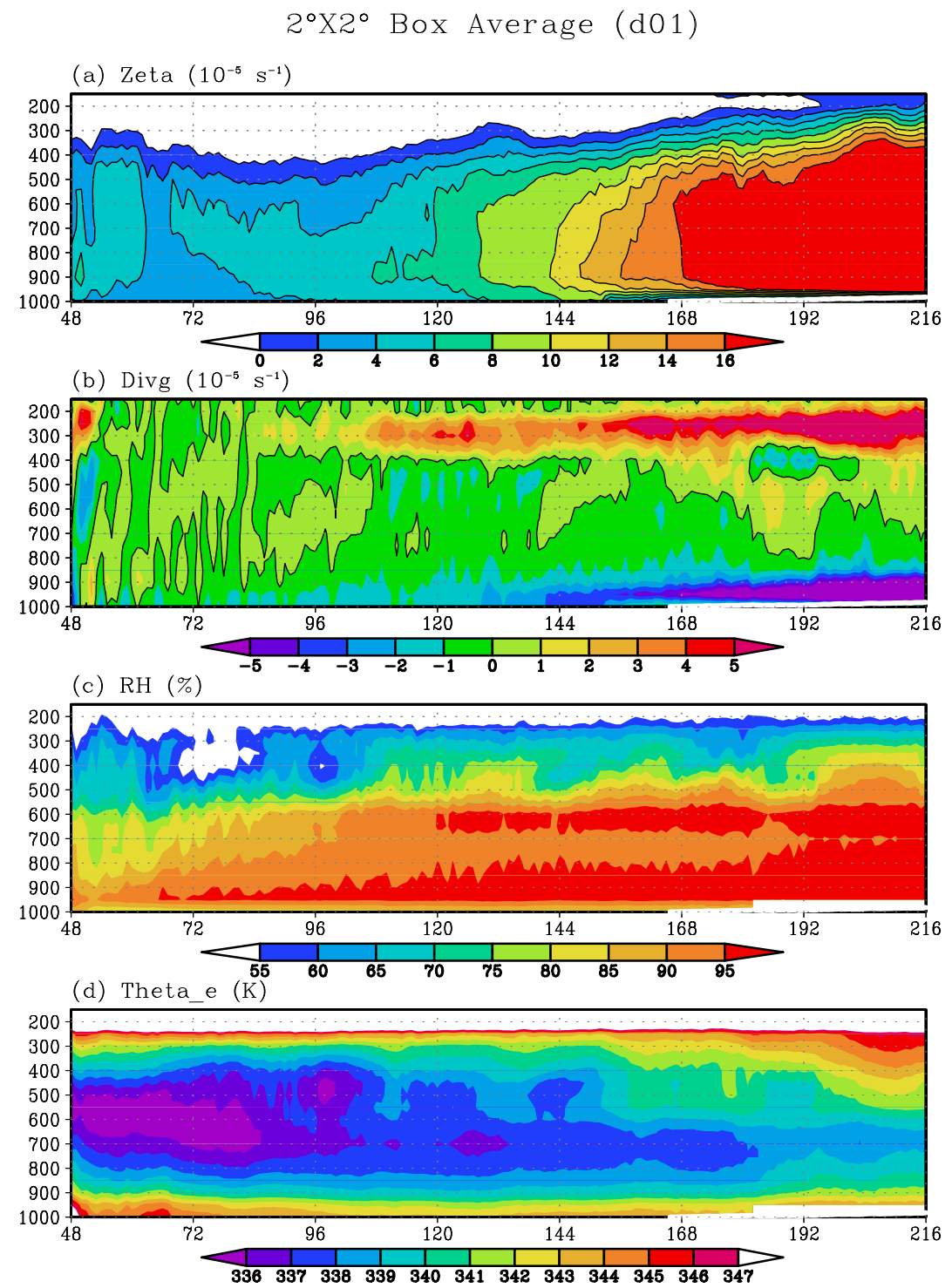

Fig. 18. Same as Fig. 16, except for the simulation with ice microphysics. See text for details.

Even with the standard WRF ice microphysics package, we do not see evidence to support the middle-level "humidity trigger" mechanism suggested by Nolan (2007). From Fig. $18 \mathrm{c}$, we see again that in the context of the tropical waveto-cyclone transition problem posed by KT81, the increase of mid-level relative humidity $(500 \mathrm{hPa} \mathrm{RH})$ does not precede the amplification of near-surface vorticity ( $900 \mathrm{hPa}$ zeta); rather, it accompanies the spin up of near-surface vorticity, and both follow the commencement of the low-level convergence $(900 \mathrm{hPa}$ Divg.) associated with the buoyant convective towers. (Some further discussion of this issue is provided in our response to an Interactive Comment.)
Finally, the time-height figure of $\theta_{e}$ (Fig. 18d) depicts an area-average surface $\theta_{e}$ that gradually decreases from $t=66 \mathrm{~h}$ to approximately $t=180 \mathrm{~h}$; the latter is well into the construction of the vorticity monolith. These results support the hypothesis that both the genesis and intensification process is not a WISHE process. The former is supported by Rotunno and Emanuel (1987) and the references cited earlier. The question of whether intensification can occur in an approximately similar manner in the absence of the WISHE intensification mechanism is answered in the affirmative elsewhere (Montgomery et al., 2010). 
(a) Stratiform Area (\%)

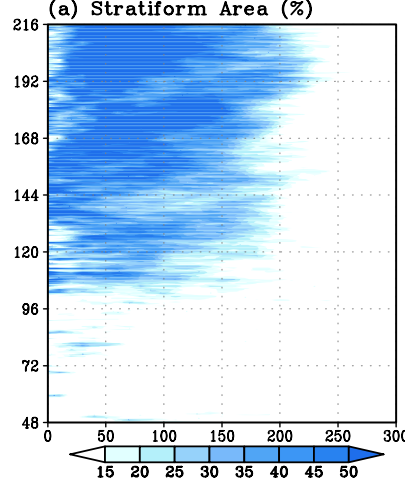

(c) Stratiform Precip $(\mathrm{mm} / \mathrm{h})$

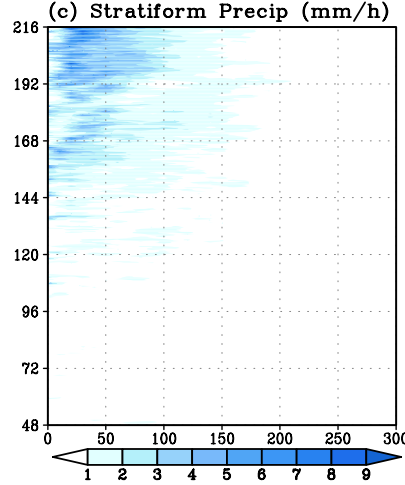

(b) Convective Area (\%)

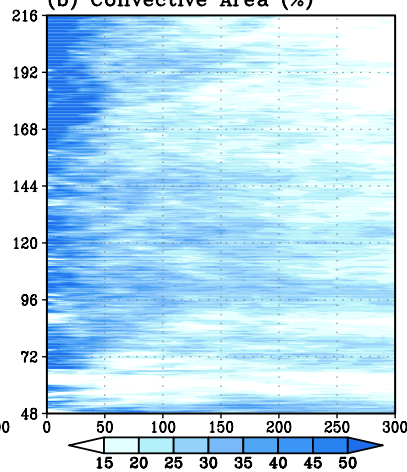

(d) Convective Precip $(\mathrm{mm} / \mathrm{h})$

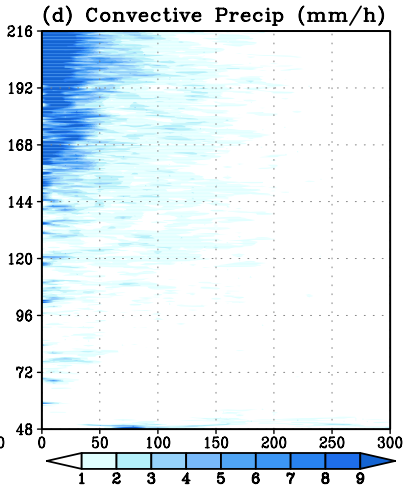

Fig. 19. Time-radial evolution of the stratiform (left) and convective (right) precipitation. The top panels show the area coverage by each rain type in percentage (\%), and the bottom panels show the azimuthal mean rain rate associated with each rain type. The ordinate is time (h) and the abscissa is radius with respect to the moving pouch center $(\mathrm{km})$.

\subsubsection{Convective versus stratiform populations within the pouch}

We address next the matter concerning the relative role of the convective and stratiform convective processes in the genesis sequence. To do this we need a simple but physically-based method for identifying (deep) convection and stratiform precipitation. Precipitation is categorized here as convective and stratiform following Tao et al. (1993) and Braun et al. (2009), and the same algorithm is used in our companion study of Wang et al. (2010a, b). First, grid points with rain rate twice as large as the average of their nearest four neighbors are identified as convective cell cores. If a grid point is designated as a convective cell core in this way, then its nearest neighbors (within one grid distance) are also designated as convective. Secondly, all grid points with surface rainfall rates greater than $25 \mathrm{~mm} \mathrm{~h}^{-1}$ are categorized as convective. To identify convective columns in which significant precipitation is not reaching the surface, columns with maximum upward vertical motions larger than $5 \mathrm{~m} \mathrm{~s}^{-1}$ or cloud liquid water below the melting level larger than $0.5 \mathrm{~g} \mathrm{~kg}^{-1}$ are designated also as convective. All remaining grid columns

whose surface precipitation is greater than $0.1 \mathrm{~mm} \mathrm{~h}^{-1}$ are categorized as stratiform; all grid columns with surface precipitation smaller than this rate are considered as nonprecipitating. Our tests of this identification algorithm show that this method is sensitive to thresholds used to distinguish the convective and stratiform rainfall. For example, if a sixteen-grid point average, instead of four-grid point average, is used to identify convective cell cores, many more grid points will be categorized as convective. To avoid overestimating the convective contribution, some of the thresholds used in this study are higher than Tao et al. (1993) or Braun et al. (2009). Our objective is to examine the quantitative evolution of stratiform and convective precipitation and their relative importance. We believe the algorithm used is adequate for this purpose.

Figure 19 shows four plots. The two along the top depict a time-radius plot of the area percentage that is stratiform and convective within the wave's pouch. The radius is defined relative to the center of the pouch and spans a domain of $300 \mathrm{~km}$ in extent. We see that the convective population is active throughout the pouch after $t=48 \mathrm{~h}$. The stratiform population on the other hand does not make a significant contribution until approximately $t=100 \mathrm{~h}$. In time, however, the convective population occupies a more substantial fraction of the area near the center of the pouch. This conclusion is confirmed in the second two figures along the bottom of the figure. The convective precipitation is maximized near the center of the pouch and progressively dominates the stratiform precipitation component.

Figure 20 shows a snapshot of the rain rate and rain type distribution from the rain+ice experiment at $t=168 \mathrm{~h}$. The top figure shows the rain rate in $\mathrm{mm} / \mathrm{h}$. The bottom figure shows the rain type. The heaviest rain rate is associated with the convective precipitation and it is spatially concentrated near the center of the pouch.

These rain plus ice results point to a containment of persistent deep convection near the center of the pouch. This containment has a very important effect in the aggregate by producing mesoscale convergence at low-levels, which leads to low-level spin up. This behavior is unlike that of stratiform convection that selectively spins up the middle levels and spins down the low-levels (Tory and Montgomery, 2006; cf. Houze, 2004).

\section{Summary and conclusions}

For tropical cyclogenesis in tropical easterly waves, the marsupial paradigm presented by DMW09 has begun to bridge the longstanding gap between observationally resolvable scales (synoptic, meso- $\alpha$ ) and infrequently and insufficiently observed meso- $\beta$ and cloud scales (meso- $\gamma$, VHTs) within and near the cyclonic trough of the parent easterly wave. Using a multiply-nested numerical model designed to test the key elements of the marsupial paradigm, we find 
(a) Rain Rate $(\mathrm{mm} / \mathrm{h})$ and $850 \mathrm{hPa}$ Streamlines (Hour 168)

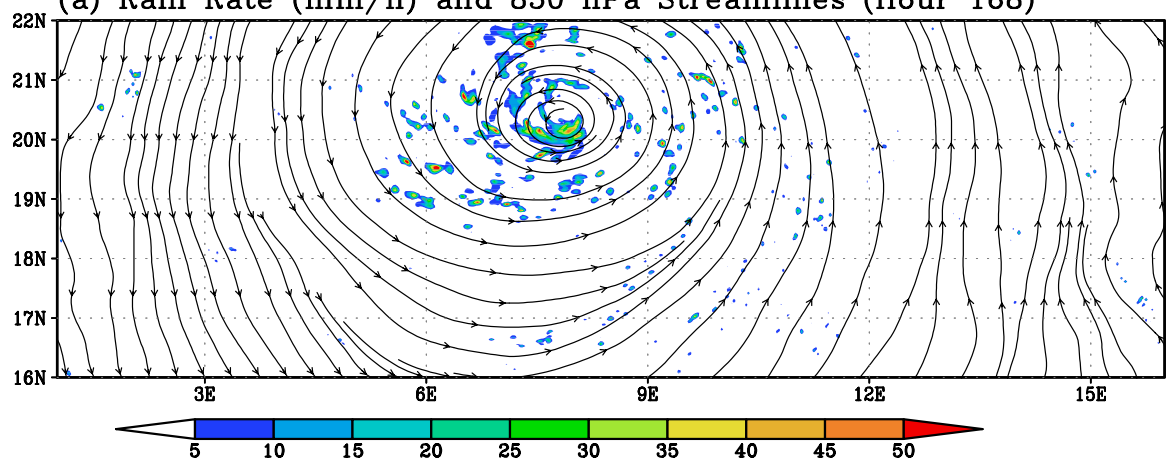

(b) Rain Type and $850 \mathrm{hPa}$ Translated Streamlines (Hour 168)

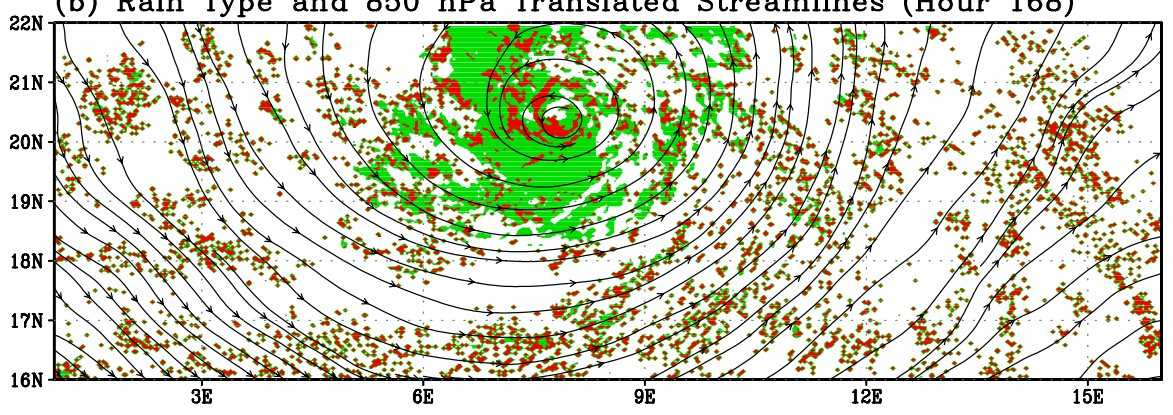

Fig. 20. Snapshot of rain rate (a) and rain type distribution (b) from the ice microphysics run at Hour 168. In (b), green shading represents stratiform precipitation and red represents convective precipitation.

broad agreement with the pioneering study of KT81. Moreover, the results suggest that the formation, aggregation and amplification of preferentially cyclonic vorticity anomalies by convective heating in the Kelvin cat's eye of a critical layer constitutes the principal pathway for tropical cyclogenesis in tropical easterly waves. Our findings directly support the first hypothesis (H1) as articulated in DMW09. This finding is demonstrated to transcend the particular horizontal resolution employed and is found to be robust to variations in cumulus parameterization, warm rain or ice microphysics formulations as represented in the standard "off-the-shelf" version of the WRF model.

As discussed in DMW09, a key concept derived from theories of the nonlinear critical layer is that the cat's eye exists prior to organization and does not depend, structurally or otherwise, on the details of organization until TC intensification begins. On the other hand, as suggested in DMW09, the moist mesoscale processes may have feedback to the synoptic-scale wave and help to maintain and possibly enhance the wave (H3). According to the second hypothesis (H2) of the marsupial paradigm, the formation of closed streamlines implies that the air within the materially closed space should be protected, to some degree, from lateral entrainment of dry air. The closure serves not only to concentrate vorticity by the aforementioned mechanism, but may account also for the accumulation of moist entropy and saturation of the tropospheric column by persistent deep moist convection in the pouch - the same convection that facilitates growth of cyclonic anomalies. This containment has a very important effect in allowing a convective type of convergence profile to dominate the closed region with a relatively smaller contribution from stratiform precipitation that is otherwise common in mesoscale convective systems (Tory and Montgomery, 2006; cf. Houze, 2004).

We find that the maximum convergence in the central region of the pouch occurs at a lower altitude than non-rotating organized tropical convection (e.g., Houze 2004), favoring rapid spin-up of one or more low-level vortices. The latter finding is consistent with Raymond and Sessions (2007) and moreover with the recent clarification of the spin up mechanisms operating in tropical cyclones by Smith et al. (2009). Using a more complete implementation of the WRF model that includes penetrative convection (hot towers, vortical or otherwise), ice microphysics and associated stratiform convection processes, the tendency for the deep convection to gradually dominate the stratiform convection near the wave's sweet spot is verified here, consistent with H2. Our results demonstrate that the marsupial sequence provides a direct "bottom-up" pathway to tropical cyclogenesis more or less independent of the mid-level transport of PV by typical mesoscale convective systems in the tropics (cf. Ritchie and Holland, 1997; Bister and Emanuel, 1997; Houze, 2004). 
In our companion study (Wang et al., 2010a, b) we have conducted a numerical study of the genesis sequence of realcase Hurricane Felix that occurred in the main development region of the tropical Atlantic during the 2007 hurricane season. A full-physics implementation of the WRF model was employed in that study (including ice microphysical processes). Felix originated from an African easterly wave, and the low-level critical layer and its associated Kelvin cat's eye formed from the interaction between the easterly wave and underlying Inter-Tropical-Convergence-Zone. As a region of closed cyclonic circulation, the cat's eye protected the moist air inside from dry air intrusion, and the formation of VHTs were favored in this vorticity- and moisture-rich environment. Similar to the current study, the cat's eye provides the focal point for the upscale aggregation of enclosed VHTs, and a tropical storm formed near the center of the cat's eye via vorticity aggregation processes.

In closing: our two complimentary numerical studies, one idealized and one real-case example, provide strong support for the marsupial paradigm. Further observational, theoretical and numerical tests are ongoing and the results of this work will be reported in due course. In addition to these research lines, a new field experiment is forthcoming to test the theory in the Atlantic sector. The experiment and its relationship with the theory discussed herein is summarized in Appendix B.

\section{Appendix A}

\section{Initialization via the reverse balance equations}

The initialization method used here follows Kurihara and Bender (1980). The horizontal primitive equations in the $\sigma$ coordinates may be written as (Phillips, 1957)

$$
\begin{aligned}
& \frac{d u}{d t}-f v=-\frac{\sigma}{\rho} \frac{\partial p_{*}}{\partial x}-\frac{\partial \Phi}{\partial x} \\
& \frac{d v}{d t}+f u=-\frac{\sigma}{\rho} \frac{\partial p_{*}}{\partial y}-\frac{\partial \Phi}{\partial y}
\end{aligned}
$$

where $\sigma=\frac{p}{p_{*}}, p_{*}$ is the surface pressure, and $\Phi$ is the geopotential. Eliminating $\rho$ using the equation of state, we have

$$
\begin{aligned}
\frac{d u}{d t}-f v & =-\frac{R T}{p_{*}} \frac{\partial p_{*}}{\partial x}-\frac{\partial \Phi}{\partial x} \\
\frac{d v}{d t}+f u & =-\frac{R T}{p_{*}} \frac{\partial p_{*}}{\partial y}-\frac{\partial \Phi}{\partial y}
\end{aligned}
$$

The geostrophic balance in the $\sigma$-coordinates becomes

$f \vec{k} \times \vec{V}+R T \nabla \operatorname{In} p_{*}+\nabla \Phi=0$

Assuming the zonal mean flow is in a state of geostrophic balance (for a flat ocean surface) we then have

$\frac{\partial \ln \bar{p}_{*}}{\partial y}=-\frac{f \bar{u}_{*}}{R \bar{T}_{*}}$ $\frac{\partial \bar{\Phi}}{\partial y}=-f \bar{u}-R \bar{T} \frac{\partial \ln \bar{p}_{*}}{\partial y}=-f\left(\bar{u}-\frac{\bar{T}}{\overline{T_{*}}} \bar{u}_{*}\right)$

where the subscript * denotes the value at the surface and the overbar denotes the zonal mean. Given $\bar{p}_{*}$ and $\bar{T}(\sigma)$ at a certain latitude $\phi_{0}$, and if $\bar{T}(\sigma)$ is approximated by $\overline{T_{0}}(\sigma)$ in the above equations, we may solve for $\bar{p}_{*}$ and $\bar{\Phi}$.

For an initial flow comprising a zonal flow plus a small, but finite amplitude easterly wave, the mass field must be obtained by solving the more general reverse balance equation in the $\sigma$-coordinates

$\nabla^{2} \Phi+\nabla\left(R T \nabla \operatorname{In} p_{*}\right)=G$

where

$G=2 J(u, v)+f \zeta-u \beta$

and where $J(u, v)=\frac{\partial u}{\partial x} \frac{\partial v}{\partial y}-\frac{\partial u}{\partial y} \frac{\partial v}{\partial x}$. The right-hand side of Eq. (A8) may be calculated from a given wind field. If, as above, $\bar{T}(\sigma)$ is approximated by its vertical profile at the center latitude $\bar{T}_{0}(\sigma)$, then simplified Poisson-type equations for the surface pressure and geopotential are obtained for $\sigma=1$ and $\sigma<1$, respectively, as follows

$$
\begin{aligned}
& \nabla^{2} I n p_{*}=\frac{G_{*}}{R T_{*_{0}}} \\
& \nabla^{2} \Phi=G-\frac{T_{0}}{T_{*_{0}}} G_{*}
\end{aligned}
$$

For an initial horizontal wind field (given $\bar{p}_{*}$ and $\bar{\Phi}$ from the geostrophic inversion Eqs. A6, A7), the total fields (the zonal mean plus perturbation) may be obtained upon solving Eqs. (A10, A11) by a relaxation method. Once $\Phi$ is determined, $T$ is then obtained from the hydrostatic relation. The temperature field in Eqs. (A10-A11) can then be updated. This procedure is repeated several times until the temperature, surface pressure and geopotential fields no longer change appreciably. The fields so constructed thus solve the reverse balance equations.

\section{Appendix B}

\section{The PREDICT (2010) experiment}

Recent years have seen several field campaigns aimed at understanding the dynamics of tropical cyclone formation. These include the Tropical Cloud Systems and Processes (TCSP) in 2005 (Halverson et al., 2007) and the NASA AMMA project in 2006. Adding in the results of earlier efforts such as the Tropical Experiment in Mexico (TEXMEX; Bister and Emanuel, 1997; Raymond et al., 1998) and even serendipitous observations of the early development of hurricane Ophelia in RAINEX (Houze et al., 2006, 2009) and occasionally from reconnaissance aircraft (Reasor et al., 2005), 
and we have a collection of studies that have sampled pieces of a large and complex scientific puzzle. As discussed in DMW09, the puzzle begins with the formation of a tropical depression, a necessary meteorological precursor that results, in most cases, in the subsequent formation of a tropical cyclone.

So why should there be a new effort? The first answer was given in the introduction to this paper: the problem of tropical depression formation remains unanswered and is one of the great remaining mysteries of the tropical atmosphere. Perhaps the greatest shortcoming of previous campaigns is the limited sampling, both in space and in time. Therefore a second answer is that it is difficult to piece together snapshots of tropical disturbances taken at different times. "Genesis" (the formation of a tropical depression-strength vortex at sub-synoptic scales) often occurs between sampling times, or after disturbances move out of range. Third, there are very few observations of both precursors to genesis and the ensuing tropical cyclone formation process. The limited range of previous projects has meant also a limited phenomenological scope, regarding precursors to genesis and the multi-scale interactions needed for TC formation. We know now that synoptic-scale precursors take many forms (tropical waves, monsoon troughs, upper-tropospheric PV features, etc.). One of the sub-hypotheses for this experiment is that the genesis process itself should be relatively invariant on mesoscale and sub-synoptic scales in spite of these differences in synoptic precursor patterns. However, we do not know if this is correct. Fourth, the marsupial paradigm of tropical cyclogenesis discussed here embodies several new, unifying hypotheses that must be tested in order to confront the issues raised above. Finally, the combination of technological resources at our disposal is unprecedented.

As discussed in DMW09 and in this paper, a key ingredient in the marsupial paradigm is the role of rotating deep moist convection and how convective organization in a rotating environment differs from that of typical mesoscale convective systems in the tropics. Differences in cloud systems arising from the unique nature of the proto-vortex environment imply a different pathway of dynamical evolution than what might otherwise occur in the absence of this environment (e.g., Figs. 16, 17, 18). The construction of a hurricane is a marvellous event, one that cannot be taken for granted in the current climate of Earth, nor in the perturbed climates to come. A unique cloud system structure and dynamical evolution, and a key role of precursor waves, contribute to this marvel in its early stages. The mesoscale aspects of this structure and evolution remain poorly understood.

To address limitations of previous campaigns, the proposed PRE-Depression Investigation of Cloud-systems in the Tropics (PREDICT) aims to dramatically increase the spatial and temporal sampling of tropical disturbances prior to, and during, genesis. The PREDICT experiment is envisioned as a close collaboration between the Unites States National Science Foundation (NSF), the National Oceanic and Atmo- spheric Administration (NOAA) and the Naval Postgraduate School in Monterey, California. The primary research tools needed are the NCAR G-V aircraft, with altitude and range advantages over previous aircraft, and two NOAA WP-3D aircraft each equipped with a tail Doppler radar, a lower fuselage (weather surveillance) radar, and the standard suite of flight-level and dropwindsonde observations that accompany normal hurricane and pre-hurricane reconnaissance. For the NCAR G-V aircraft, we propose to double-crew the aircraft for a portion of the field phase to allow sampling disturbances for as much as 16 out of $24 \mathrm{~h}$. The project will cover the majority of the Atlantic sector, including the Caribbean, and therefore will be poised to observe many forms of precursor disturbances and be positioned to uncover the common physical processes of genesis on the mesoscale. Finally, the project aims to fully integrate the plethora of satellite data and derived products now available, coordinating the aircraft missions to maximize the total data coverage and physical interpretation.

Acknowledgements. We appreciate the enthusiastic reception of our colleagues attending the NASA AMMA/TCSP workshop in Baltimore (May 2007) where an overview of some of this work was first presented by MTM. Helpful comments on this work were provided by our NPS/NRL-Monterey colleagues. We thank C. Davis, L. Bosart and A. Heymsfeld for their collaboration in the proposed PREDICT experiment and their assistance with the writing of Appendix B, and thank Roger Smith and Kevin Tory for their helpful comments. This research was supported by the National Aeronautics and Space Administration, MIPR NNG07HU171 and Contract NNH04CC63C, the National Science Foundation Grants ATM-0733380, ATM-0715426, ATM-0649944, ATM-0649946, ATM-0227632, the Office of Naval Research grant N001408WR20129 and the U.S. Naval Postgraduate School in Monterey, California.

Edited by: H. Wernli

\section{References}

Bister, M. and Emanuel, K. A.: The genesis of Hurricane Guillermo: TEXMEX analyses and a modeling study, Mon. Weather Rev., 125, 2662-2682, 1997.

Bui, H. H., Smith, R. K., Montgomery, M. T., and Peng, J.: Balanced and unbalanced aspects of tropical cyclone intensification, Q. J. Roy. Meteorol. Soc., 135, 1715-1731, 2009.

Braun, S. A., Montgomery, M. T., Mallen, K. J., and Reasor, P. D.: Simulation and interpretation of the genesis of tropical storm GERT (2005) as part of the NASA tropical cloud systems, J. Atmos. Sci., 67, 999-1025, 2010.

Cram, T. A., Montgomery, M. T., and Hertenstein, R. F. A.: Early Evolution of Vertical Vorticity in a Numerically Simulated Idealized Convective Line, J. Atmos. Sci., 59, 2113-2127, 2002.

Davis, C. A. and Trier, S. B.: Cloud-Resolving Simulations of Mesoscale Vortex Intensification and Its Effect on a Serial Mesoscale Convective System. Mon. Wea. Rev., 130, 28392858, 2002. 
Dunkerton, T. J., Montgomery, M. T., and Wang, Z.: Tropical cyclogenesis in a tropical wave critical layer: easterly waves, Atmos. Chem. Phys., 9, 5587-5646, 2009, http://www.atmos-chem-phys.net/9/5587/2009/.

Emanuel, K. A., Neelin, J. D., and Bretherton, C. S.: On largescale circulations in convecting atmosphere, Qu. J. Roy. Meteor. Soc., 120, 1111-1143, 1994.

Emanuel, K. A.: Divine Wind: The History and Science of Hurricanes, Oxford University Press, New York, USA, 285 pp., 2005.

Eliassen, A. and Lystad, M.: The Ekman layer of a circular vortex: A numerical and theoretical study, Geophys. Norv., 31, 1-16, 1977.

Guinn, T. A. and Schubert, W. H.: Hurricane spiral bands, J. Atmos. Sci., 50, 3380-3403, 1993.

Hall, N. M. J., Kiladis, G. N., and Thorncroft, C. D.: Threedimensional structure and dynamics of African easterly waves. Part II: dynamical modes, J. Atmos. Sci., 63, 2231-2245, 2006.

Halverson, J., Black, M., Braun, S., et al.: NASA's Tropical Cloud Systems and Processes Experiment, B. Am. Meteor. Soc., 88, 867-882, 2007.

Haynes, P. H. and McIntyre, M. E.: On the evolution of vorticity and potential vorticity in the presence of diabatic heating and frictional or other forces, J. Atmos. Sci., 44, 828-841, 1987.

Holton, J. R.: An Introduction to Dynamic Meteorology, 3rd edn., Academic Press, San Diego, 511 pp., 1992.

Hong, S. -Y. and Lim, J.-O.: The WRF Single-moment 6-class microphysics scheme (WSM6), J. Korean. Meteor. Soc., 42, 129$151,2006$.

Houze Jr., R. A.: Mesoscale convective systems, Rev. Geophys., 42, RG4003, doi:10.1029/2004RG000150, 2004.

Houze, R. A., Lee, W.-C., and Bell, M. M.: Convective Contribution to the Genesis of Hurricane Ophelia (2005), Mon. Weather Rev., 137, 2778-2800, 2009.

Kurihara, Y. and Bender, M. A.: Use of a Movable Nested-Mesh Model for Tracking a Small Vortex, Mon. Weather Rev., 108, 1792-1809, 1980.

Kurihara, Y. and Tuleya, R. E.: A Numerical Simulation Study on the Genesis of a Tropical Storm, Mon. Weather Rev., 109, 16291653, 1981.

Marín, J. C., Raymond, D. J., and Raga, G. B.: Intensification of tropical cyclones in the GFS model, Atmos. Chem. Phys., 9, 1407-1417, doi:10.5194/acp-9-1407-2009, 2009.

McWilliams, J. C.: The emergence of isolated coherent vortices in turbulent flow, J. Fluid Mech., 140, 21-43, 1984.

Montgomery, M. T. and Enagonio, J.: Tropical Cyclogenesis via Convectively Forced Vortex Rossby Waves in a ThreeDimensional Quasigeostrophic Model, J. Atmos. Sci., 55, 31763207, 1998.

Montgomery, M. T., Nicholls, M. E., Cram, T. A., and Saunders, A. B.: A vortical hot tower route to tropical cyclogenesis, J. Atmos. Sci., 63, 355-386, 2006.

Montgomery, M. T., Nguyen, V. S., Smith, R. K., and Persing, J.: Do tropical cyclones intensify by WISHE?, Q. J. Roy. Meteorol. Soc., 135, 1697-1714, 2010.

Molinari, J., Vollaro, D., and Corbosiero, K. L.: Tropical cyclone formation in a sheared environment: a case study, J. Atmos. Sci., 61, 2493-2509, 2004.

Nolan, D. S.: What is the trigger for tropical cyclogenesis?, Aust.
Meteorol. Mag., 56, 241-266, 2007.

Phillips, N. A.: A coordinate system having some special advantages for numerical forecasting, J. Meteorol., 14, 184-185, 1957.

Raymond, D. J., López-Carrillo, C., and López Cavazos, L.: Casestudies of developing east Pacific easterly waves, Q. J. Roy. Meteorol. Soc., 124, 2005-2034, 1998.

Raymond, D. J. and Sessions, S. L.: Evolution of convection during tropical cyclogenesis, Geophys. Res. Lett., 34, L06811, doi:10.1029/2006GL028607, 2007.

Reasor, P. D., Montgomery, M. T., and Boast, L. F.: Mesoscale observations of the genesis of Hurricane Dolly (1996), J. Atmos. Sci., 62, 3151-3171, 2005.

Ritchie, E. A. and Holland, G. J.: Scale interactions during the formation of Typhoon Irving, Mon. Weather Rev., 125, 1377-1396, 1997.

Rotunno, R. and Emanuel, K. A.: An Air-Sea Interaction Theory for Tropical Cyclones. Part II: Evolutionary Study Using a Nonhydrostatic Axisymmetric Numerical Model, J. Atmos. Sci., 44, 542-561, 1987.

Skamarock, W. C., Klemp, J. B., Dudhia, J., Gill, D. O., Barker, D. M., Wang, W., and Powers, J. G.: A description of the Advanced Research WRF Version 2. NCAR Tech. Note/TN468+STR, 88 pp., 2005.

Smith, R. K., Montgomery, M. T., and Nguyen, V. S.: Tropical cyclone spin up revisited, Q. J. Roy. Meteorol. Soc., 135, 13211335, 2009.

Srivastava, R.: A model of intense downdrafts driven by the melting and evaporation of precipitation, J. Atmos. Sci., 44, 1752-1773, 1987.

Tao, W. K., Simpson, J., Sui, C. H., Ferrier, B., Lang, S., Scala, J., Chou, M. D., and Pickering, K.: Heating, Moisture, and Water Budgets of Tropical and Midlatitude Squall Lines: Comparisons and Sensitivity to Longwave Radiation, J. Atmos. Sci., 50, 673690, 1993.

Thorncroft, C. D. and Hoskins, B. J.: An idealized study of African easterly waves. I: a linear view, Q. J. Roy. Meteorol. Soc., 120, 953-982, 1994.

Tory, K. J. and Montgomery, M. T.: Internal influences on tropical cyclone formation. Topic 2.2 in Sixth International Workshop on Tropical Cyclones, San Jose, Costa Rica, World Meteorological Organization, 22 pp., 2006.

Tory, K. J., Montgomery, M. T., and Davidson, N. E.: Prediction and diagnosis of tropical cyclone formation in an NWP system. Part I: the critical role of vortex enhancement in deep convection, J. Atmos. Sci., 63, 3077-3090, 2006 a.

Tory, K. J., Montgomery, M. T., Davidson, N. E., and Kepert, J. E.: Prediction and diagnosis of tropical cyclone formation in a NWP system. Part II: a diagnosis of tropical cyclone Chris formation, J. Atmos. Sci., 63, 3091-3113, 2006 b.

Tory, K. J., Davidson, N. E., and Montgomery, M. T.: Prediction and diagnosis of tropical cyclone formation in an NWP system. Part III: diagnosis of developing and non-developing storms, J. Atmos. Sci., 64, 3195-3213, 2007.

Tory, K. J. and Montgomery, M. T.: Tropical cyclone formation: A synopsis of the internal dynamics, AMS 28th Conference on Hurricanes and Tropical Meteorology, Orlando, FL, 2008. 
Wang, Z., Montgomery, M. T., and Dunkerton, T. J.: Genesis of Pre-hurricane Felix (2007). Part I: The Role of the Easterly Wave Critical Layer, J. Atmos. Sci., 67, 1711-1729, 2010.

Wang, Z., Montgomery, M. T., and Dunkerton, T. J.: Genesis of Prehurricane Felix (2007). Part II: Warm core formation, precipitation evolution and predictability, J. Atmos. Sci., 67, 1730-1744, 2010 .
Weisman, M. L. and Davis, C. A.: Mechanisms for the Generation of Mesoscale Vortices within Quasi-Linear Convective Systems, J. Atmos. Sci., 55, 2603-2622, 1998. 